\title{
CONIVEAU SPECTRAL SEQUENCES OF CLASSIFYING SPACES FOR EXCEPTIONAL AND SPIN GROUPS
}

\author{
M. KAMEKO, M. TEZUKA, AND N. YAGITA
}

\begin{abstract}
Let $k$ be an algebraically closed field of $\operatorname{ch}(k)=0$ and $G$ be a simple simply connected algebraic group $G$ over $k$. By using results of cohomological invariants, we compute the coniveau spectral sequence for classifying spaces $B G$.
\end{abstract}

\section{INTRODUCTION}

Let $G$ be a simple simply connected algebraic group over an algebraically closed field $k$ in $\mathbb{C}$. The cohomological invariant $\operatorname{Inv}^{*}(G ; \mathbb{Z} / p)$ is (roughly speaking) the ring of natural maps $H^{1}(F ; G) \rightarrow H^{*}(F ; \mathbb{Z} / p)$ for finitely generated field $F$ over $k$. (For detailed definition and properties, see the book [Ga-Me-Se] by Garibaldi, Merkurjev and Serre.)

Let $B G$ be the classifying space of $G$. Totaro showed that

$$
\operatorname{Inv}^{*}(G ; \mathbb{Z} / p) \cong H^{0}\left(B G ; H_{\mathbb{Z} / p}^{*}\right)
$$

where $H^{*}\left(X ; H_{\mathbb{Z} / p}^{*^{\prime}}\right)$ is the cohomology of the Zarisky sheaf induced from the presheaf $H_{e t}^{*}(V ; \mathbb{Z} / p)$ for open subsets $V$ of $X$. This sheaf cohomology is also the $E_{2}$-term

$$
E_{2}^{*, *^{\prime}} \cong H^{*}\left(B G ; H_{\mathbb{Z} / p}^{*^{\prime}}\right) \Longrightarrow H^{*}(B G ; \mathbb{Z} / p)
$$

of the coniveau spectral sequence by Bloch-Ogus [Bl-Og].

We restrict to consider a group $G$ such that it has only one conjugacy class of nontoral maximal elementary abeilan $p$-group $A$. For exceptional cases, $G=G_{2}, F_{4}, E_{6}$ for $p=2, G=F_{4}, E_{6}, E_{7}$ for $p=3$, and $G=E_{8}$ for $p=5$. We also consider groups $\operatorname{Spin}_{n}, n \geq 7$.

Let $W_{G}(A)$ be the Weyl group of $G$ for $A$. Then by using Rost, Serre and Garibaldi's results [Ga], we easily see that

$$
\operatorname{Res}_{\text {Inv }}: \operatorname{Inv}^{*}(G ; \mathbb{Z} / p) \cong \operatorname{Inv}^{*}(A ; \mathbb{Z} / p)^{W_{G}(A)}
$$

1991 Mathematics Subject Classification. Primary 11E72, 12G05, 55P35; Secondary $55 \mathrm{~T} 25,57 \mathrm{~T} 05$.

Key words and phrases. coniveau spectral sequence, motivic cohomology, exceptional groups. 
for cases of the above groups except for $\left(E_{6}, p=2\right)$ and $\left(\operatorname{Spin}_{n}, p=2\right)$, $n \geq 10$.

Let $Q_{i}$ be the Milnor operation and let

$$
Q(n)=\Lambda\left(Q_{0}, \ldots, Q_{n}\right) .
$$

We easily see that operations $Q_{i}$ can extend on $H^{*}\left(B G ; H_{\mathbb{Z} / p}^{*}\right)$ for these fields $k$. In particular, $H^{*}\left(B A ; H_{\mathbb{Z} / p}^{*^{\prime}}\right)^{W_{G}(A)}$ has also the $Q(\infty)$ module structure. We can prove that for the above cases except for $\left(E_{7}, p=3\right)$, the invariant is generated as $Q(\infty)$-algebras by elements in $\operatorname{Res}\left(\operatorname{Inv}^{*^{\prime}}(G ; \mathbb{Z} / p)\right)$ and $\operatorname{Res}\left(H^{*}\left(B G ; H_{\mathbb{Z} / p}^{*}\right)\right)=\operatorname{Res}\left(C H^{*}(B G) / p\right)$.

(Moreover it is a direct sum of free $Q(n)$-modules.)

These facts imply the following theorem.

Theorem 1.1. Let $G=G_{2}, \operatorname{Spin}_{n}(7 \leq n \leq 9), F_{4}$ for $p=2, G=$ $F_{4}, E_{6}$ for $p=3$, or $G=E_{8}, p=5$. Then the following restriction map

$$
\operatorname{Res}_{E_{2}}: H^{*}\left(B G ; H_{\mathbb{Z} / p}^{*^{\prime}}\right) \rightarrow H^{*}\left(B A ; H_{\mathbb{Z} / p}^{*^{\prime}}\right)^{W_{G}(A)}
$$

is an epimorphism.

For $\left(E_{7}, p=3\right)$, the map $\operatorname{Res}_{E_{2}}$ is not epic, while $\operatorname{Res}_{I n v}$ is epic.

We note that the restriction map

$$
\operatorname{Res}_{H \mathbb{Z} / p}: H^{*}(B G ; \mathbb{Z} / p) \rightarrow H^{*}(B A ; \mathbb{Z} / p)^{W_{G}(A)}
$$

is not an epimorphism for $p \geq 3$, while $H^{*}(B A ; \mathbb{Z} / p) \cong H^{*}\left(B A ; H_{\mathbb{Z} / p}^{*^{\prime}}\right)$ as algebras. (Indeed, $B G$ is 3 -connected but $H^{0}\left(B G ; H_{\mathbb{Z} / p}^{3}\right) \neq 0$.) Note also that the right hand side invariant and $\operatorname{Im}\left(\operatorname{Res}_{H \mathbb{Z} / p}\right)$ are computed by Kameko-Mimura [Ka-Mi] for odd primes $p$.

When $p=2$, the maps $\operatorname{Res}_{H \mathbb{Z} / 2}$ are even isomorphic except for the case $E_{6}$. However note $\left.([\mathrm{Or}-\mathrm{Vi}-\mathrm{Vo}])\right)$

$$
H^{*}\left(B A ; H_{\mathbb{Z} / 2}^{*^{\prime}}\right) \cong g r H^{*}(B A ; \mathbb{Z} / 2) \cong \mathbb{Z} / 2\left[y_{1}, \ldots, y_{n}\right] \otimes \Lambda\left(x_{1}, \ldots, x_{n}\right)
$$

with $\beta x_{i}=y_{i}$ but $y_{i} \neq x_{i}^{2}=0$ as the cases $p=o d d$. So we know

$$
\operatorname{Res}_{H \mathbb{Z} / 2} \cong \operatorname{gr}\left(H^{*}(B A ; \mathbb{Z} / 2)^{W_{G}(A)}\right) \stackrel{\subset}{\neq} H^{*}\left(B A ; H_{\mathbb{Z} / 2}^{*^{\prime}}\right)^{W_{G}(A)}
$$

for the above groups.

The arguments seem something subtle and we give here an example, the case $G=G_{2}$ and $p=2$. Then $A \cong(\mathbb{Z} / 2)^{3}$ and $W_{G}(A) \cong$ $G L_{3}(\mathbb{Z} / 2)$, moreover

$$
H^{*}\left(B G_{2} ; \mathbb{Z} / 2\right) \cong H^{*}(B A ; \mathbb{Z} / 2)^{W_{G}(A)} \cong \mathbb{Z} / 2\left[w_{4}, w_{6}, w_{7}\right] \quad\left|w_{i}\right|=i .
$$

The cohomological invariant is known by Rost and Serre

$$
\operatorname{Inv}^{*}\left(G_{2} ; \mathbb{Z} / 2\right) \cong \mathbb{Z} / 2\left\{1, u_{3}\right\} \quad\left|u_{3}\right|=3
$$


where $u_{3}=x_{1} x_{2} x_{3}$ in $H^{0}\left(B A ; H_{\mathbb{Z} / 2}^{3}\right)$. From Mui and Kameko-Mimura results $[\mathrm{Ka}-\mathrm{Mi}]$, we can show

$$
H^{*}\left(B A ; H_{\mathbb{Z} / 2}^{*^{\prime}}\right)^{W_{G}(A)} \cong \mathbb{Z} / 2\left[c_{4}, c_{6}\right] \otimes\left(\mathbb{Z} / 2\{1\} \oplus \mathbb{Z} / 2\left[c_{7}\right] \otimes Q(2)\left\{u_{3}\right\}\right)
$$

where $Q_{0} Q_{1} Q_{2}\left(u_{3}\right)=c_{7}, \operatorname{deg}\left(c_{i}\right)=(i, i)\left(\right.$ and $w_{i}^{2}=c_{i}$ in $\left.H^{*}(B A ; \mathbb{Z} / 2)\right)$. These $c_{i}$ are represented by Chern classes, and hence $R e s_{E_{2}}$ is an epimorphism.

Of course $u_{3} \notin R e s_{H \mathbb{Z} / 2}$, and moreover we see

$$
H^{*}\left(B A ; H_{\mathbb{Z} / 2}^{*^{\prime}}\right)^{W_{G}(A)} / \operatorname{Res}_{H \mathbb{Z} / 2} \cong \mathbb{Z} / 2\left[c_{4}, c_{6}\right]\left\{u_{3}\right\} .
$$

For example, we have

$$
Q_{0}\left(u_{3}\right)=w_{4}, \quad Q_{1}\left(u_{3}\right)=w_{6}, \quad Q_{0} Q_{1}\left(u_{3}\right)=w_{7}, \quad Q_{2}\left(u_{3}\right)=w_{4} w_{6} .
$$

Here $u_{3}$ does not exist in $H^{*}\left(B G_{2} ; \mathbb{Z} / 2\right)$, and hence $d_{r}\left(u_{3}\right)=y \neq 0$ for some $r \geq 2$ and $y \in H^{*}\left(B G_{2} ; H_{\mathbb{Z} / 2}^{*}\right)$ in the coniveau spectral sequence. We can see this $r=2$.

Theorem 1.2. We have the epimorphism (as bidegree $Q(2)$-modules) from $H^{*}\left(B G_{2} ; H_{\mathbb{Z} / 2}^{*^{\prime}}\right)$ onto

$$
\begin{gathered}
H^{*}\left(B A ; H_{\mathbb{Z} / 2}^{*^{\prime}}\right)^{W_{G}(A)} \oplus\left(H^{*}\left(B A ; H_{\mathbb{Z} / 2}^{*^{\prime}}\right)^{W_{G}(A)} / \operatorname{Res}_{H \mathbb{Z} / 2}\right)(-1)[2] \\
\cong \mathbb{Z} / 2\left[c_{4}, c_{6}\right] \otimes\left(\mathbb{Z} / 2\{1, y\} \oplus \mathbb{Z} / 2\left[c_{7}\right] \otimes Q(2)\left\{u_{3}\right\}\right)
\end{gathered}
$$

where $(-1)[2]$ is the degree shift operation so that $\operatorname{deg}(y)=(2,2)$. Moreover $d_{2}\left(u_{3}\right)=y$ in the coniveau spectral sequence.

Moreover if the Gottlieb transfer exists in the motivic cohomology, then the above epimorphism is indeed isomorphism. The similar fact also holds for $G=\operatorname{Spin}_{7}$ and $p=2$.

Note that $y$ in the above theorem, is a $(\bmod (p))$ Griffith element, namely,

$$
y \in K \text { Ker }\left(\text { cycle map }: C H^{*}(B G) / p \rightarrow H^{2 *}(B G ; \mathbb{Z} / p)\right) .
$$

Each non zero element in $\left(H^{*}\left(B A ; H_{\mathbb{Z} / p}^{*^{\prime}}\right)^{W_{G}(A)} / \operatorname{Res}_{H \mathbb{Z} / p}\right)$ of $\operatorname{deg}=(*-$ $2, *+1)$ corresponds to a Griffith element of $\operatorname{deg}=(*, *)$. So we can construct many Griffith elements in $C H^{*}(B G) / p$ for the above groups $G$.

An outline of this paper is following. In $\S 2$, we recall the relation between the motivic cohomology $H^{*, *^{\prime}}(X ; \mathbb{Z} / p)$ and the sheaf cohomology $H^{*}\left(X ; H_{\mathbb{Z} / p}^{*^{\prime}}\right)$. In $\S 3$, we show that $H^{*}\left(X ; H_{\mathbb{Z} / p}^{*^{\prime}}\right)$ has the $Q_{i}$-action. In $\S 4$, we recall the cohomological invariant and give a sufficient condition such that $\operatorname{Res}_{E_{2}}$ is epic when $\operatorname{Inv}^{*}(G ; \mathbb{Z} / p)$ is known. In $\S 5$, we study the Dickson invariant for $H^{*}\left(B A ; H_{\mathbb{Z} / p}^{*^{\prime}}\right)$ using $Q_{i}$ actions by KamekoMimura. In $\S 6-\S 8$, we compute $H^{*}\left(B G ; H_{\mathbb{Z} / p}^{*^{\prime}}\right)$ for concrete cases, 
e.g., $\left(G_{2}, p=2\right)$ is studied in $\S 6$. In $\S 9$, we study the relation between $H^{*}\left(B G ; H_{\mathbb{Z} / p}^{*^{\prime}}\right)$ and the Brown-Peterson theory $B P^{*}(B G)$. In the last section, we study the image of Griffith elements to $B P^{*}(B G) \otimes_{B P^{*}} \mathbb{Z} / p$, in particular, for $\left(\operatorname{Spin}_{9}, p=2\right)$.

\section{MOTIVIC COHOMOLOGY}

Let $X$ be a smooth (quasi projective) variety over a field $k \subset \mathbb{C}$. Let $H^{*, *^{\prime}}(X ; \mathbb{Z} / p)$ be the $\bmod (p)$ motivic cohomology defined by Voevodsky and Suslin ([Vo1-3]).

Recall that the $(\bmod p) B(n, p)$ condition holds if

$$
H^{m, n}(X ; \mathbb{Z} / p) \cong H_{e t}^{m}\left(X ; \mu_{p}^{\otimes n}\right) \quad \text { for all } m \leq n .
$$

Recently M.Rost and V.Voevodsky ([Vo5],[Su-Jo],[Ro]) proved that $B(n, p)$ condition holds for each $p$ and $n$. Hence the Bloch-Kato conjecture also holds. Therefore in this paper, we always assume the $B(n, p)$-condition and also the Bloch-Kato conjecture for all $n, p$.

Moreover we always assume that $k$ contains a primitive $p$-th root of unity. For these cases, we see the isomorphism $H_{e t}^{m}\left(X ; \mu_{p}^{\otimes n}\right) \cong$ $H_{e t}^{m}(X ; \mathbb{Z} / p)$. Let $\tau$ be a generator of $H^{0,1}(\operatorname{Spec}(k) ; \mathbb{Z} / p) \cong \mathbb{Z} / p$, so that

$$
\operatorname{colim}_{i} \tau^{i} H^{*, *^{\prime}}(X ; \mathbb{Z} / p) \cong H_{e t}^{*}(X ; \mathbb{Z} / p) .
$$

Let $H^{*}\left(X ; H_{\mathbb{Z} / p}^{*^{\prime}}\right)$ be the sheaf cohomology where $H_{\mathbb{Z} / p}^{n}$ is the Zarisky sheaf induced from the presheaf $H_{e t}^{n}(V ; \mathbb{Z} / p)$ for open subset $V$ of $X$.

Let $X=\bigcup U_{\lambda}$ for Zarisky open sets $U_{\lambda}$. The sheaf cohomology $H^{*}\left(X ; H_{\mathbb{Z} / p}^{*^{\prime}}\right)$ is defined as the colimit of the cohomology of the following $\check{C}$ eck complex

$$
\begin{aligned}
(2.1) & \rightarrow \prod \Gamma_{\left(i_{1}, \ldots, i_{n}\right)} \stackrel{\delta}{\rightarrow} \prod \Gamma_{\left(j_{1}, \ldots, j_{n+1}\right)} \rightarrow \\
\text { where } \Gamma_{\left(i_{1}, \ldots, i_{n}\right)} & =\Gamma\left(U_{i_{1}} \cap \ldots \cap U_{i_{n}} ; H^{*}\left(U_{i_{1}} \cap \ldots \cap U_{i_{n}} ; \mathbb{Z} / p\right)^{a}\right)
\end{aligned}
$$

and where $H^{*}(-; \mathbb{Z} / p)^{a}$ is a sheaficication of the presheaf $H^{*}(-; \mathbb{Z} / p)$. Here $\delta$ is induced map from the inclusions $U_{i} \cap U_{j} \subset U_{i}, U_{i} \cap U_{j} \subset U_{j}$.

The Beilinson and Lichtenbaum conjecture ( hence $B(n, p)$-condition ) (see $\left.\left[V_{0} 2,5\right]\right)$ implies the exact sequences of cohomology theories

Theorem 2.1. ([Or-Vi-Vo], [Vo5]) There is a long exact sequence

$$
\begin{aligned}
\rightarrow H^{m, n-1}(X ; \mathbb{Z} / p) & \stackrel{\times \tau}{\rightarrow} H^{m, n}(X ; \mathbb{Z} / p) \\
& \rightarrow H^{m-n}\left(X ; H_{\mathbb{Z} / p}^{n}\right) \rightarrow H^{m+1, n-1}(X ; \mathbb{Z} / p) \stackrel{\times \tau}{\rightarrow} .
\end{aligned}
$$

In particular, we have 
Corollary 2.2. We have the additive isomorphism

$$
H^{m-n}\left(X ; H_{\mathbb{Z} / p}^{n}\right) \cong H^{m, n}(X ; \mathbb{Z} / p) /(\tau) \oplus \operatorname{Ker}(\tau) \mid H^{m+1, n-1}(X ; \mathbb{Z} / p)
$$

where $H^{m, n}(X ; \mathbb{Z} / p) /(\tau)=H^{m, n}(X ; \mathbb{Z} / p) /\left(\tau H^{m, n-1}(X ; \mathbb{Z} / p)\right)$.

Note that the long exact sequence in Theorem 2.1 induces the $\tau$ Bockstein spectral sequence

$$
E(\tau)_{1}=H^{m-n}\left(X ; H_{\mathbb{Z} / p}^{n}\right) \Longrightarrow \operatorname{colim}_{i} \tau^{i} H^{*, *^{\prime}}(X ; \mathbb{Z} / p) \cong H_{e t}^{*}(X ; \mathbb{Z} / p) .
$$

On the other hand, the filtration coniveau is given by

$$
N^{c} H_{e t}^{m}(X ; \mathbb{Z} / p)=\cup_{Z} \operatorname{Ker}\left\{H_{e t}^{m}(X ; \mathbb{Z} / p) \rightarrow H_{e t}^{m}(X-Z ; \mathbb{Z} / p)\right\}
$$

where $Z$ runs in the set of closed subschemes of $X$ of codim $=c$. The induced spectral sequence is called the coniveau spectral sequence. Bloch-Ogus [Bl-Og] proved that its $E_{2}$-term is given by

$$
E(c)_{2}^{c, m-c} \cong H^{c}\left(X, H_{\mathbb{Z} / p}^{m-c}\right) .
$$

By Deligne ( foot note (1) in Remark 6.4 in [Bl-Og]) and Paranjape (Corollary $4.4 \mathrm{in}[\mathrm{Pj}]$ ), it is proven that there is an isomorphism of the coniveau spectral sequence with the Leray spectral sequence for the natural map of the sites. Hence we have ;

Theorem 2.3. (Deligne, Parajape) There is the isomorphism $E(c)_{r}^{c, m-c} \cong$ $E(\tau)_{r-1}^{m, m-c}$ for $r \geq 2$ of spectral sequences. Hence the filtrations are the same $N^{c} H_{e t}^{m}(X ; \mathbb{Z} / p)=F_{\tau}^{m, m-c}$ where

$$
F_{\tau}^{m, m-c}=\operatorname{Im}\left(\times \tau^{c}: H^{m \cdot m-c}(X ; \mathbb{Z} / p) \rightarrow H^{m, m}(X ; \mathbb{Z} / p)\right) .
$$

\section{COHOMOLOGY OPERATION}

Let $t_{\mathbb{C}}: H^{*, *^{\prime}}(X ; \mathbb{Z} / p) \rightarrow H^{*}(X(\mathbb{C}) ; \mathbb{Z} / p)$ be the realization map ([Vo1]) for the inclusion $k \subset \mathbb{C}$. The motivic cohomology has (Bockstein, reduced powered) cohomology operations ([Vo2,4])

$$
\begin{gathered}
\beta: H^{*, *^{\prime}}(X ; \mathbb{Z} / p) \rightarrow H^{*+1, *^{\prime}}(X ; \mathbb{Z} / p) \\
P^{i}: H^{*, *^{\prime}}(X ; \mathbb{Z} / p) \rightarrow H^{*+2 i(p-1), *^{\prime}+i(p-1)}(X ; \mathbb{Z} / p)
\end{gathered}
$$

which are compatible with the usual (topological) cohomology operations by the realization map $t_{\mathbb{C}}$. Voevodsky defines the Milnor operation $Q_{i}$ also in the mod $p$ motivic cohomology

$$
Q_{i}: H^{*, *^{\prime}}(-; \mathbb{Z} / p) \rightarrow H^{*+2 p^{i}-1, *^{\prime}+p^{i}-1}(-; \mathbb{Z} / p) .
$$

Here we define the weight degree by

$$
w(x)=2 n-m\left(\text { resp. }=n^{\prime}-m^{\prime}\right)
$$


for $0 \neq x \in H^{m, n}(X ; \mathbb{Z} / p)$ (resp. $H^{m^{\prime}}\left(X ; H_{\mathbb{Z} / p}^{n^{\prime}}\right)$ ). Similarly, we also define the weight degree for cohomology operations and differentials of spectral sequences,e.g.,

$$
w(\tau)=2, \quad w\left(P^{i}\right)=0, \quad w\left(Q_{j}\right)=-1 .
$$

Let $\rho_{p}=\left(\xi_{p}\right) \in k^{*} /\left(k^{*}\right)^{p}=H^{1,1}(\operatorname{Spec}(k) ; \mathbb{Z} / p)$ where $\xi_{p}$ is the primitive $p$-th root of unity. The $Q_{i}$ operation has the same property as the topological case only with $\bmod \left(\rho_{2}\right)$. For example, $Q_{i}$ is a derivative only $\bmod \left(\rho_{2}\right)$.

Let $A_{p}$ be the mod $p$ Steenrod algebra generated by all cohomology operations on $H^{*, *^{\prime}}(X ; \mathbb{Z} / p)$. ( Voevodsky proved that $A_{p}$ is multiplicatively generated by elements in $H^{*, *^{\prime}}(\operatorname{Spec}(k) ; \mathbb{Z} / p), P^{j}$ and $Q_{i}$.)

Lemma 3.1. Suppose $\rho_{p}=0$. Then the Steenrod algebra $A_{p}$ acts on the etale cohomolgy $H^{*}(X ; \mathbb{Z} / p)$.

Proof. In $H^{*, *^{\prime}}(\operatorname{Spec}(k) ; \mathbb{Z} / p)$, we know

$$
P^{i}(\tau)=0 \text { for } i>0, \text { and } \beta(\tau)=\rho_{p}=0 .
$$

When $p \geq 3$, the Cartan formula holds in the motivic cohomology (Proposition 9.6 in [Vo4]), and we have

$$
P^{i}(\tau x)=\tau P^{i}(x) \text { for } i>0, \text { and } \beta(\tau x)=\tau \beta(x) .
$$

From the $B(n, p)$ condition, $H_{e t}^{*}(X ; \mathbb{Z} / p)=\operatorname{colim}_{i} \tau^{i} H^{*, *^{\prime}}(X ; \mathbb{Z} / p)$, which implies the lemma.

For $p=2$, we also know from Proposition 9.6 in [Vo4],

$$
\begin{aligned}
S q^{2 *}(x y) & =\sum_{i} S q^{2 i}(x) S q^{2 *-2 i}(y)+\tau \sum_{i} S q^{2 i+1}(x) S q^{2 *-2 i-1}(y), \\
S q^{2 *+1}(x y) & =\sum_{j} S q^{j}(x) S q^{2 *+1-j}(y)+\rho_{2} \sum_{i} S q^{2 i+1}(x) S q^{2 *-2 i-1}(y) .
\end{aligned}
$$

Since $\rho_{2}=0$, we see $S q^{2 i+1}(\tau)=0$, and so $S q^{*}(\tau x)=\tau S q^{*}(x)$. This also induces the lemma.

Theorem 3.2. Suppose $\rho_{p}=0$. Then the cohomology operation $Q_{i}$ and $P^{i}$ can be extended on the $\tau$-Bockstein spectral sequence and so on the coniveau spectral sequence $E_{r}, r \geq 2$ (e.g., on $H^{*}\left(X ; H_{\mathbb{Z} / p}^{*^{\prime}}\right)$ ).

Proof. In the stable $\mathbb{A}^{1}$-homotopy category $S H$ ot, let $H \mathbb{Z} / p$ be the Eilenberg-MacLane spectrum representing the mod $p$ motivic cohomology

$$
H^{*, *^{\prime}}(X ; \mathbb{Z} / p) \cong \operatorname{Hom}_{S H o t}\left(X, S^{*, *^{\prime}} \wedge H \mathbb{Z} / p\right)
$$

where $S^{*, *^{\prime}}$ is the sphere of bidegree $\left(*, *^{\prime}\right)$. 
Let $o p .=Q_{i}$ or $P^{i}$ of bidegree $(m, n)$. Consider the diagram

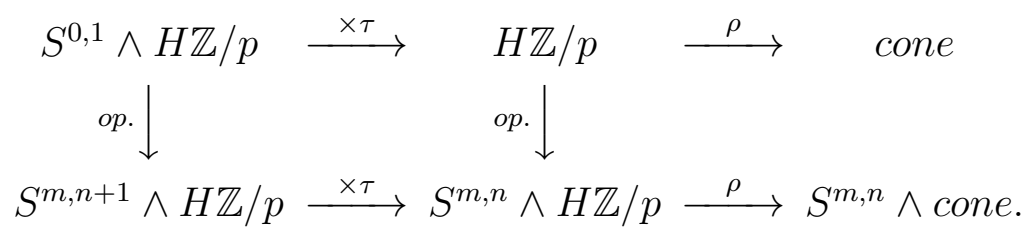

Here cone is the mapping cone of $\tau$ so that

$$
H^{*+*^{\prime}}\left(X ; H_{\mathbb{Z} / p}^{*^{\prime}}\right) \cong H_{\text {om }} \text { SHot }\left(X, S^{*, *^{\prime}} \wedge \text { cone }\right) .
$$

In the above diagram, we see $\rho \cdot o p . \tau=0$. Hence there is a map $o p^{\prime}$. : cone $\rightarrow S^{m, n} \wedge$ cone such that $\rho \cdot o p .=o p^{\prime} . \cdot \rho$.

Here we do not see yet that $A_{p}$ acts on $E_{r}$, e.g., we do not see that $Q_{i}$ generates the exterior algebra $Q(\infty)$. However when $r=2$, the following theorem holds.

Lemma 3.3. Let $k$ be an algebraically closed field. Then the Steenrod algebra $A_{p}$ acts on $H^{*}\left(X ; H_{\mathbb{Z} / p)}^{*^{\prime}}\right)$.

Proof. Recall that $H^{*}\left(X ; H_{\mathbb{Z} / p}^{*^{\prime}}\right)$ is defined as the cohomology of the $\check{C}$ eck complex. Given op. $\in A_{p}$, by the universality of sheaficication, the following diagram from $(2.1)$ is commutative

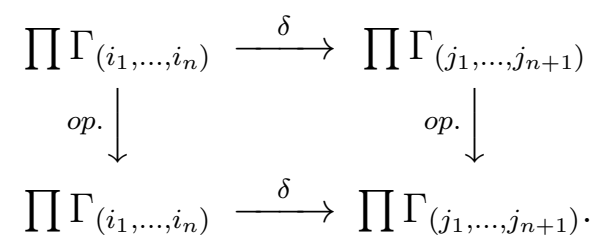

Thus we have the desired result.

Let us write $H^{*, *^{\prime}}=H^{*, *^{\prime}}(\operatorname{Spec}(k) ; \mathbb{Z} / p)$ and $H^{*}=K_{M}^{*}(k) / p$ so that $H^{*, *^{\prime}} \cong H^{*}[\tau]$. (Note if $k$ is algebraically closed, $H^{*, *^{\prime}} \cong \mathbb{Z} / p[\tau]$.) For an elementary abelian $p$-group $A=A_{n} \cong(\mathbb{Z} / p)^{n}$, the $\bmod (p)$ motivic cohomology is given by Voevodsky $\left(\left[\mathrm{Vo}_{2}, 4\right]\right)$

$$
H^{*, *^{\prime}}(B A ; \mathbb{Z} / p) \cong H^{*, *^{\prime}}\left[y_{1}, \ldots, y_{n}\right] \otimes \Delta\left(x_{1}, . ., x_{n}\right)
$$

with $x_{i}^{2}=y \tau+x \rho_{2}$ for $p=2$ and $x_{i}^{2}=0$ otherwise.

Since $\operatorname{Ker}(\tau) \mid H^{*, *^{\prime}}(B A ; \mathbb{Z} / p)=0$, from Corollary 2.2, we have

$$
\begin{gathered}
H^{*}\left(B A ; H_{\mathbb{Z} / p}^{*^{\prime}}\right) \cong H^{*, *}(B A ; \mathbb{Z} / p) /\left(\tau H^{*, *-1}(B A ; \mathbb{Z} / p)\right) \\
\cong H^{*}\left[y_{1}, \ldots, y_{n}\right] \otimes \Lambda\left(x_{1}, \ldots, x_{n}\right) \quad\left(\bmod \left(\rho_{2}\right)\right)
\end{gathered}
$$

for all primes $p$. Each $Q_{i}$ is a derivation $\bmod \left(\rho_{2}\right)$, and hence

$$
Q_{0} \ldots Q_{s-1}\left(x_{1} \ldots x_{s}\right)=\sum \operatorname{sgn}\left(j_{1}, \ldots, j_{s}\right) y_{1}^{p^{j_{1}}} y_{2}^{p_{2}} \ldots y_{s}^{p^{j_{s}}} \neq 0 \quad \bmod \left(\rho_{2}\right)
$$


where $\left(j_{1}, \ldots, j_{s}\right)$ are permutations of $(0, \ldots, s-1)$.

Let us write

$$
\begin{gathered}
Q(n)=\Lambda\left(Q_{0}, \ldots, Q_{n}\right), \\
\bar{Q}(n)=Q(n)-\mathbb{Z} / p\left\{Q_{0} \ldots Q_{n}\right\}=\mathbb{Z} / p\left\{Q_{i_{0}} \ldots Q_{i_{s}} \mid 0 \leq i_{k} \leq n, s<n\right\} .
\end{gathered}
$$

Let $u_{i}=x_{1} \ldots x_{i} \in H^{0}\left(B A ; H_{\mathbb{Z} / p}^{i}\right)$. For example, we have

$$
\begin{gathered}
H^{*}\left(B A ; H_{\mathbb{Z} / p}^{*^{\prime}}\right) \supset H^{*}\left[y_{1}, \ldots, y_{n}\right] \otimes\left(\oplus_{i} \bar{Q}(i-1)\left\{u_{i}\right\}\right) \\
\supset \oplus_{i} H^{*}\left[y_{1}, \ldots, y_{i}\right] \otimes Q(i-1)\left\{u_{i}\right\} \quad(*)
\end{gathered}
$$

since $Q_{0} \ldots Q_{i-1}\left(u_{i}\right) \in H^{*}\left[y_{1}, \ldots, y_{i}\right]\left\{y_{1} \ldots y_{i}\right\}$. In sections bellow, we show that the last sum $(*)$ of free $Q(i-1)$-modules contains $H^{*}\left(B G ; H_{\mathbb{Z} / p}^{*^{\prime}}\right)^{W_{G}(A)}$, as a direct summand, for many cases of $G$. (See Assumption (1) in $\S 4$.

\section{COHOMOLOGICAL INVARIANT}

Let $G$ be a linear algebraic group over $k$. Recall that $H^{1}(k ; G)$ is the first non abelian Galois cohomology set of $G$, which represents the set of $G$-torsors over $k$. The cohomology invariant is defined by

$$
\operatorname{Inv}^{i}(G, \mathbb{Z} / p)=\operatorname{Func}\left(H^{1}(F ; G) \rightarrow H^{i}(F ; \mathbb{Z} / p)\right)
$$

where Func means natural functions for each field $F$ which is finitely generated over $k$. (For details for the definition or properties, see the book [Ga-Me-Se].)

Totaro proved [Ga-Me-Se] the following theorem in the letter to Serre.

Theorem 4.1. (Totaro) $\operatorname{Inv}^{*}(G ; \mathbb{Z} / p) \cong H^{0}\left(B G ; H_{\mathbb{Z} / p}^{*}\right)$.

Hereafter (throughout this paper), we assume that $k$ is an algebraically closed field in $\mathbb{C}$. Moreover, in this paper, we only consider simple simply connected groups $G$ which have the following property. we assume that the algebraic group $G$ has only one conjugacy class $A$ of non toral maximal elementary abelian $p$-subgroups. Exceptional groups are

$$
G=\left\{\begin{array}{l}
G_{2}, F_{4}, E_{6} \quad \text { for } p=2 \\
F_{4}, E_{6}, E_{7} \quad \text { for } p=3 \\
E_{8} \quad \text { for } p=5 .
\end{array}\right.
$$

For spin groups $\operatorname{Spin}_{n}$, we consider the cases $n \leq 9$ only in this paper.

We consider the restriction maps (of cohomology) to $A$ and the maximal torus $T_{G}$

$$
\begin{gathered}
\operatorname{Res}_{H \mathbb{Z} / p}: H^{*}(B G ; \mathbb{Z} / p) \stackrel{i^{*}}{\rightarrow} H^{*}\left(B T_{G} ; \mathbb{Z} / p\right) \times H^{*}(B A ; \mathbb{Z} / p) \\
\stackrel{p r \cdot}{\rightarrow} H^{*}(B A ; \mathbb{Z} / p)^{W_{G}(A)} .
\end{gathered}
$$


By the Quillen's theorem the above $i^{*}$ has nilpotent kernel. More strongly, Toda, Kono, Tezuka and Kameko show $i^{*}$ is really injective, namely, $H^{*}(B G ; \mathbb{Z} / p)$ is detected by $A$ and $T_{G}$. Moreover when $p=2$, $\operatorname{Res}_{H \mathbb{Z} / 2}$ are isomorphic except the case $E_{6}$. However $R e s_{H \mathbb{Z} / p}$ is not epic for $p \geq 3$.

On the other hand, by Serre, Rost and Garibaldi([Ga-Me-Se],[Ga]), $\operatorname{Inv}^{*}(G ; \mathbb{Z} / p)$ are computed for these groups, e.g.,

$$
\operatorname{Inv}^{*}(G ; \mathbb{Z} / p) \cong\left\{\begin{array}{l}
\mathbb{Z} / p\left\{1, u_{3}\right\} \quad \text { for }\left(G_{2}, E_{6}, p=2\right),\left(F_{4}, E_{7}, p=3\right), \\
\quad\left(E_{8}, p=5\right) \\
\mathbb{Z} / p\left\{1, u_{3}, u_{4}\right\} \quad \text { for }\left(\operatorname{Spin}_{7}, p=2\right),\left(E_{6}, p=3\right) \\
\mathbb{Z} / p\left\{1, u_{3}, u_{4}, u_{5}\right\} \quad \text { for }\left(\operatorname{Spin}_{9}, p=2\right) \\
\mathbb{Z} / p\left\{1, u_{3}, u_{4}, u_{4}^{\prime}, u_{5}\right\} \quad \text { for }\left(\operatorname{Spin}_{8}, p=2\right) \\
\mathbb{Z} / p\left\{1, u_{3}, u_{5}\right\} \quad \text { for }\left(F_{4}, p=2\right) .
\end{array}\right.
$$

(Moreover Rost and Garibaldi determined $\operatorname{Inv}^{*}\left(\operatorname{Spin}_{n} ; \mathbb{Z} / 2\right)$ for $n \leq$ $12)$.

For these groups, we note (Ga-Me-Se],[Ga]) the the restriction

$$
\operatorname{Res}_{\text {Inv }}: \operatorname{Inv}^{*}(G ; \mathbb{Z} / p) \rightarrow \operatorname{Inv}^{*}(A ; \mathbb{Z} / p) \cong \Lambda\left(x_{1}, \ldots, x_{n}\right) .
$$

is injective (identifying $u_{i}=x_{1} \ldots x_{i}$ and $u_{4}^{\prime}=x_{1} x_{2} x_{3} x_{5}$ ). We will show the following theorem in $\S 6-8$ bellow (by computations of concrete cases)

Theorem 4.2. Let $G$ be an above type except for $G=E_{6}$ and $p=2$. Then

$$
\operatorname{Res}_{\text {Inv }}: \operatorname{Inv}^{*}(G ; \mathbb{Z} / p) \cong \operatorname{Inv}^{*}(A ; \mathbb{Z} / 2)^{W_{G}(A)} .
$$

Remark. When $G=E_{6}$ and $p=2$, the above $\operatorname{Res}_{I n v}$ is not epic. We want to extend above isomorphism in the theorem to say that

$$
\operatorname{Res}_{E_{2}}: H^{*}\left(B G ; H_{\mathbb{Z} / p}^{*^{\prime}}\right) \rightarrow H^{*}\left(B A ; H_{\mathbb{Z} / p}^{*^{\prime}}\right)^{W_{G}(A)}
$$

is an epimorphism. (Of course for $p \geq 3$ the above map is not injective.) We will prove the following assumption (in the sections bellow) for the above groups except for $\left(E_{6}, p=2\right)$ and $\left(E_{7}, p=3\right)$. (When $G=\operatorname{Spin}_{8}$, some modification of Assumption (1) holds.)

Assumption When $\operatorname{Inv}^{*}(G ; \mathbb{Z} / p) \cong \mathbb{Z} / p\left\{1, u_{i_{1}}, \ldots, u_{i_{m}}\right\}$, there is a bidegree isomorphism

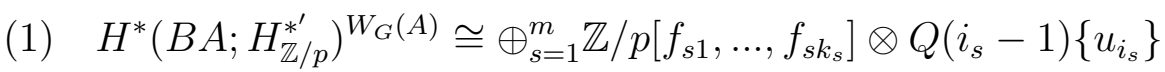

(2) $f_{s t} \in \operatorname{Res}_{H \mathbb{Z} / p}\left(H^{2 * * *}(B G ; \mathbb{Z} / p)\right)=\operatorname{Res}\left(C H^{*}(B G) / p\right)$

$$
\text { for all } 1 \leq s \leq m, 1 \leq t \leq k_{s} \text {. }
$$


If this assumption is satisfied then $H^{*}\left(B A ; H^{*^{\prime}}\right)^{W_{G}(A)}$ is generated as bidegree $Q(\infty)$-algebra by $u_{i_{s}}$ and $\operatorname{Res}\left(C H^{*}(B G) / p\right)$. Hence the surjectivity of $\operatorname{Res}_{E_{2}}$ is immediate.

Lemma 4.3. If Assumption (1),(2) are satisfied, then

$$
\operatorname{Res}_{E_{2}}: H^{*}\left(B G ; H_{\mathbb{Z} / p}^{*^{\prime}}\right) \rightarrow H^{*}\left(B A ; H_{\mathbb{Z} / p}^{*^{\prime}}\right)^{W_{G}(A)}
$$

is an epimorphism.

Thus we can prove Theorem 1.1 in the introduction. As for the statements of differential and (Griffith elements), the following lemma is useful.

Lemma 4.4. Let $\operatorname{Res}_{\text {Inv }}(a) \neq 0$ for $a \in \operatorname{Inv}^{i}(G ; \mathbb{Z} / p)=H^{0}\left(B G ; H_{\mathbb{Z} / p}^{*}\right)$. (Namely, the above element is a permanent cycle in the coniveau spectral sequence.) Moreover let

$$
Q_{j_{1}} \ldots Q_{j_{i-3}}(a) \notin H^{*}(B G ; \mathbb{Z} / p) .
$$

Then $d_{2}(a)=y \neq 0 \in H^{2}\left(B G ; H_{\mathbb{Z} / p}^{i-1}\right)$ in the coniveau spectral sequence, and elements

$$
Q_{j_{1}} \ldots Q_{j_{i-3}}(y) \neq 0 \in C H^{*}(B G) / p=H^{2 * *}(B G ; \mathbb{Z} / p)
$$

are Griffith elements (i.e., in the kernel of $C H^{2 *}(B G) / p \rightarrow H_{\text {et }}^{2 *}(B G ; \mathbb{Z} / p)$ ).

Proof. Take $q=Q_{j_{1}} \ldots Q_{j_{i-3}}(a)$. Since $q$ does not exist in $H^{*}(B G ; \mathbb{Z} / p)$, we see $d_{r}(q) \neq 0$ in the spectral sequence for some $r$.

This $r=2$ because the following reason of weight degree. First note

$$
w\left(d_{r}\right)=w t(1,1-r)=2(1-r)-1=1-2 r .
$$

Since $w(q)=w(a)-(i-3)=3$, we have

$$
w\left(d_{r}(q)\right)=3+1-2 r=4-2 r .
$$

If $r \geq 3$, then the above weight is negative and $d_{r}(q)=0$.

This implies that $d_{2}(a) \neq 0$. Otherwise

$$
d_{2}(q)=d_{2}\left(Q_{j_{1}} \ldots Q_{j_{i-3}} a\right)=Q_{j_{1} \ldots Q_{j_{n-3}}}\left(d_{2}(a)\right)=0,
$$

which is a contradiction.

\section{DICKSON INVARIANT}

At first we assume $p \geq 3$. Dickson computed the ring of invariants of $\mathbb{Z} / p\left[y_{1}, \ldots, y_{n}\right]$ with respect to the action of $G L_{n}(\mathbb{Z} / p)$. The ring of invariants is a polynomial algebra

$$
D_{n}=\mathbb{Z} / p\left[y_{1}, \ldots, y_{n}\right]^{G L_{n}(\mathbb{Z} / p)} \cong \mathbb{Z} / p\left[c_{n, 0}, \ldots, c_{n, n-1}\right]
$$


where the generators are given by the equation

$$
\mathcal{O}_{n}(X)=\prod_{y \in \mathbb{Z} / p\left\{y_{1}, \cdots, y_{n}\right\}}(X+y)=X^{p^{n}}+\sum_{j=0}^{n-1}(-1)^{n-j} c_{n, j} X^{p^{j}}
$$

Let reg: $A \rightarrow G L_{n}(\mathbb{C})$ be the regular representation and $c(r e g)$ the total Chern class. Then it is well known that

$$
c(\text { reg })=\mathcal{O}_{n}(1)=1-c_{n, n-1}+\ldots+(-1)^{n} c_{n, 0} .
$$

We also note the following lemma.

Lemma 5.1. (Lemma 2.3,2.4 in [Ka-Ya2]) Let $\rho: A_{n} \rightarrow G L_{m}(\mathbb{C})$ be an representation such that $c(\rho) \in H^{*}(B A ; \mathbb{Z} / p)^{S L_{n}(\mathbb{Z} / p)}$. Then $c(\rho)=$ $c(r e g)^{a}$ for some $a \geq 0$.

For the invariant ring $S D_{n}$ under $S L_{n}(\mathbb{Z} / p)$, we have

$$
\begin{gathered}
S D_{n}=\mathbb{Z} / p\left[y_{1}, \ldots, y_{n}\right]^{S L_{n}(\mathbb{Z} / p)} \\
\cong D_{n}\left\{1, e_{n}, \ldots, e_{n}^{p-2}\right\} \quad \text { with } e_{n}^{p-1}=c_{n, 0} \\
\cong D_{n}^{\prime} \otimes \mathbb{Z} / p\left[e_{n}\right] \quad \text { with } D_{n}^{\prime}=\mathbb{Z} / p\left[c_{n, 1}, \ldots, c_{n, n-1}\right] .
\end{gathered}
$$

Mui computed the ring of invariants of

$$
H^{*}\left(B A ; H_{\mathbb{Z} / p}^{*^{\prime}}\right) \cong \mathbb{Z} / p\left[y_{1}, \ldots, y_{n}\right] \otimes \Lambda\left(x_{1}, \ldots x_{n}\right)
$$

with respect to the action of $S L_{n}(\mathbb{Z} / p)$. (In fact, Mui studied $H^{*}(B A ; \mathbb{Z} / p)$ for odd prime $p$, however we study $H^{*}\left(B A ; H_{\mathbb{Z} / p}^{*^{\prime}}\right)$ for all primes.) Of course $u_{n}=x_{1} \ldots x_{n}$ is invariant under $S L_{n}(\mathbb{Z} / p)$. In terms of Milnor's operation, we may state Mui's result in the following form.

Theorem 5.2. (Mui[Mu], Kameko-Mimura [Ka-Mi])

$$
\begin{aligned}
H^{*}\left(B A ; H_{\mathbb{Z} / p}^{*^{\prime}}\right)^{S L_{n}(\mathbb{Z} / p)} \cong \mathbb{Z} / p\left[e_{n}, c_{n, 1}, \ldots, c_{n, n-1}\right] \otimes\left(\mathbb{Z} / p\{1\} \oplus \bar{Q}(n-1)\left\{u_{n}\right\}\right) \\
\simeq D_{n}^{\prime} \oplus S D_{n} \otimes Q(n-1)\left\{u_{n}\right\}
\end{aligned}
$$

where $Q_{0} \ldots Q_{n-1} u_{n}=e_{n}$.

The $Q_{i}$-operation acts on $u_{n}$ as follows.

Lemma 5.3. (Kameko-Mimura [Ka-Mi], $[K a-Y a 1])$ For $x \in H^{*}\left(B A ; H_{\mathbb{Z} / p}^{*^{\prime}}\right)$, it holds

$$
\left(Q_{n}+\sum_{i=0}^{n-1}(-1)^{n-i} c_{n, i} Q_{i}\right)(x)=0, \quad Q_{0} \ldots \hat{Q}_{i} \ldots Q_{n}\left(u_{n}\right)=c_{n, i} e_{n} .
$$

Let $U_{n} \subset S L_{n}(\mathbb{Z} / p)$ be the maximal unipotent subgroup generated by upper triangular matrices with diagonals 1 , so that $U_{n}$ is a Sylow $p$-subgroup of $S L_{n}(\mathbb{Z} / p)$. The invariant under this group is given by Mui, Kameko-Mimura. 
Theorem 5.4. (Kameko-Mimura Theorem 4.2 in [Ka-Mi]) Let $G^{\prime} \subset$ $G L_{n}(\mathbb{Z} / p)$ such that $\mathbb{Z} / p\left[y_{1}, \ldots, y_{n}\right]^{G^{\prime}} \cong \mathbb{Z} / p\left[f_{1}, \ldots, f_{n}\right]$ and

$$
H^{*}\left(B A_{n} ; H_{\mathbb{Z} / p}^{*^{\prime}}\right)^{G^{\prime}} \cong \mathbb{Z} / p\left[f_{1}, \ldots, f_{n}\right]\left\{v_{1}=1, \ldots, v_{2^{n}}\right\} .
$$

Then the invariant under $G=\left\langle G^{\prime}, U_{n+1}\right\rangle \subset G L_{n+1}(\mathbb{Z} / p)$ is given by

(1) $\mathbb{Z} / p\left[y_{1}, \ldots, y_{n+1}\right]^{G} \cong \mathbb{Z} / p\left[f_{1}, \ldots, f_{n}, \mathcal{O}_{n}\left(y_{n+1}\right)\right]$

(2) $H^{*}\left(B A_{n+1} ; H_{\mathbb{Z} / p}^{*^{\prime}}\right)^{G} \cong$

$$
\mathbb{Z} / p\left[f_{1}, \ldots, f_{n}, \mathcal{O}_{n}\left(y_{n+1}\right)\right] \otimes\left(\mathbb{Z} / p\left\{v_{1}, \ldots, v_{2^{n}}\right\} \oplus Q(n-1)\left\{u_{n+1}\right\}\right) .
$$

\section{Corollary 5.5.}

$$
H^{*}\left(B A ; H_{\mathbb{Z} / p}^{*^{\prime}}\right)^{U_{n}} \cong \oplus_{i=0}^{n} \mathbb{Z} / p\left[\mathcal{O}_{0}\left(y_{1}\right), \ldots, \mathcal{O}_{i-1}\left(y_{i}\right)\right] \otimes Q(i-1)\left\{u_{i}\right\} .
$$

Corollary 5.6. (Lemma 5.8 in $[\mathrm{Ka}-\mathrm{Ya}]$ )

$$
\mathcal{O}\left(y_{n+1}\right) u_{n}=\left(Q_{n}+\sum_{i=0}^{n-1}(-1)^{n-i} c_{n, i} Q_{i}\right)\left(u_{n+1}\right) .
$$

Hereafter this section, we assume $p=2$. Of course we have the isomorphism $H^{*}(B A ; \mathbb{Z} / 2) \cong \mathbb{Z} / 2\left[x_{1}, \ldots, x_{n}\right]$ and its invariant under $G L_{n}(\mathbb{Z} / 2)=S L_{n}(\mathbb{Z} / 2)$ is

$$
\mathbb{Z} / 2\left[x_{1}, \ldots, x_{n}\right]^{G L_{n}(\mathbb{Z} / 2)} \cong \mathbb{Z} / 2\left[d_{n, 0}, \ldots, d_{n, n-1}\right]
$$

where the generators are given by the equation

$$
\mathcal{O}_{n}^{\prime}(X)=\prod_{x \in \mathbb{Z} / p\left\{x_{1}, \cdots, x_{n}\right\}}(X+x)=X^{2^{n}}+\sum_{j=0}^{n-1} d_{n, j} X^{2^{j}} .
$$

Here $d_{n, i}^{2}=c_{n, i}$ in $H^{*}(B A ; \mathbb{Z} / 2)$ identifying $y_{i}=x_{i}^{2}$. The Milnor $Q_{i^{-}}$ operations (see (2.6) in Schuster-Yagita [Sc-Ya]) are given as the case $p$ odd. (Hereafter let us write $d_{n, i}$ by $d_{i}$ simply.)

$$
d_{0}=Q_{0} \ldots Q_{n-2}\left(u_{n}\right), \quad d_{i}=Q_{0} \ldots \hat{Q}_{i-1} \ldots Q_{n-1}\left(u_{n}\right) / d_{0} .
$$

From Lemma 2.1 in [Sc-Ya], we have

$$
(*) \quad Q_{n-1}\left(d_{i}\right)=d_{0} d_{i}, \quad Q_{i-1}\left(d_{i}\right)=d_{0} .
$$

In $H^{*}\left(B A ; H_{\mathbb{Z} / 2}^{*^{\prime}}\right)$, we can get more strong result. Let us write simply

$$
\begin{gathered}
I\left(G L_{n}\right)=\operatorname{gr}\left(H^{*}\left(B A_{n} ; \mathbb{Z} / 2\right)^{G L_{n}(\mathbb{Z} / 2)}\right) \subset H^{*}\left(B A ; H_{\mathbb{Z} / 2}^{*^{\prime}}\right) \\
\operatorname{Igr}\left(G L_{n}\right)=H^{*}\left(B A_{n} ; H_{\mathbb{Z} / 2}^{*^{\prime}}\right)^{G L_{n}(\mathbb{Z} / 2) .}
\end{gathered}
$$

By Kameko-Mimura theorem, we have showed

$$
\left.\operatorname{Igr}\left(G L_{n}\right) \cong D_{n}^{\prime} \oplus D_{n} \otimes Q(n-1)\left\{u_{n}\right\}\right)
$$

where $D_{n}=\mathbb{Z} / 2\left[c_{n, n-1}, \ldots, c_{n, 0}\right] \quad$ and $\quad D_{n}^{\prime}=\mathbb{Z} / 2\left[c_{n, n-1}, \ldots, c_{n, 1}\right]$. 
Lemma 5.7. In $H^{*}\left(B A ; H_{\mathbb{Z} / 2}^{*^{\prime}}\right)$, we have

$$
\begin{gathered}
d_{i}=Q_{0} \ldots \hat{Q}_{i-1} \ldots \hat{Q}_{n-1}\left(u_{n}\right), \quad d_{i} d_{0}=Q_{0} \ldots \hat{Q}_{i-1} \ldots Q_{n-1}\left(u_{n}\right) . \\
d_{i} d_{j}=Q_{0} \ldots \hat{Q}_{i-1} \ldots \hat{Q}_{j-1} \ldots Q_{n-1}\left(u_{n}\right) \quad i \neq j .
\end{gathered}
$$

Proof. Consider the element

$$
a_{i}=Q_{0} \ldots \hat{Q}_{i-1} \ldots Q_{n-2} \hat{Q}_{n-1}\left(u_{n}\right) \in \operatorname{Igr}\left(G L_{n}\right) .
$$

Then $Q_{i-1}\left(a_{i}\right)=d_{0}$ and $Q_{n-1} a_{i}=d_{0} d_{i}$.

Using property $(*)$, we see $Q_{j}\left(a_{i}-d_{i}\right)=0$ for all $j$. Of course $a_{i}-d_{i} \in \operatorname{Igr}\left(G L_{n}\right)$. From Kameko-Mimura theorem, we still know

$$
\operatorname{Igr}\left(G L_{n}\right) \cap \cap_{j} \operatorname{Ker}\left(Q_{j}\right)=D_{n} .
$$

This means $d_{i}=a_{i} \in \operatorname{Igr}\left(G L_{n}\right)$. (In fact $d_{i}=a_{i} \bmod \left(D_{n}\right)$ in $H^{*}\left(B A_{n} ; \mathbb{Z} / 2\right)$, but $d_{i}=a_{i}$ exactly in $H^{*}\left(B A_{n} ; H_{\mathbb{Z} / 2}^{*^{\prime}}\right)$.)

Therefore we have

$$
d_{i}=Q_{0} \ldots \hat{Q}_{i-1} \ldots \hat{Q}_{n-1}\left(u_{n}\right), \quad d_{i} d_{0}=Q_{0} \ldots \hat{Q}_{i-1} \ldots Q_{n-1}\left(u_{n}\right) .
$$

By the similar arguments, we have

$$
d_{i} d_{j}=Q_{0} \ldots \hat{Q}_{i-1} \ldots \hat{Q}_{j-1} \ldots Q_{n-1}\left(u_{n}\right) .
$$

Of course $I\left(G L_{n}\right) \subset \operatorname{Igr}\left(G L_{n}\right)$, but this injection is not an isomorphism for $n \geq 3$.

Lemma 5.8. Let $n \geq 3$. Then we have

$$
\operatorname{Igr}\left(G L_{n}\right) / I\left(G L_{n}\right) \supset Q(n-1) /\left(Q(n-1)^{+}\right)^{n-2}\left\{u_{n}\right\} .
$$

Proof. Consider the element

$$
x=Q_{0} \ldots \hat{Q}_{i-1} \ldots \hat{Q}_{j-1} \ldots \hat{Q}_{k-1} \ldots Q_{n-1}\left(u_{n}\right) .
$$

Its image $Q_{i-1}(x)=d_{j} d_{k}$ or $d_{j}$ (when $k=n$.) Hence $x$ is not in $I\left(G L_{n}\right)$ because $Q_{i}$ maps $n$-product elements into also $n$-product elements. (If $x \in I\left(G L_{n}\right)$, then $x$ must be a sum of $d_{i}$ or $d_{i} d_{j}$, but it still appeared in $\left(Q(n-1)^{+}\right)^{n-2}\left(u_{n}\right)$.) Thus we get the lemma.

Let $A=A_{n}$ be a maximal elementary abelian $p$-subgroup of $G$ and $W_{G}(A)$ its Weyl group.

Lemma 5.9. If $\operatorname{Res}_{\text {Inv }}: \operatorname{Inv}^{*}(G ; \mathbb{Z} / p) \rightarrow \operatorname{Inv}^{*}\left(A_{n} ; \mathbb{Z} / p\right)^{W_{G}(A)}$ is an epimorphism, then

$$
\begin{aligned}
& \left(Q(n-1)^{+}\right)^{n-2}\left\{u_{n}\right\} \subset \operatorname{Res}_{H_{\mathbb{Z} / p}}\left(H^{*}(B G ; \mathbb{Z} / p) \rightarrow H^{*}\left(B A_{n} ; \mathbb{Z} / p\right)\right) \\
& \text { (e.g., } \left.d_{1}, \ldots, d_{n-1} \text { for } p=2 \text { are in } \operatorname{Res}_{H_{\mathbb{Z} / 2}}\right) .
\end{aligned}
$$


Proof. Let $x$ be an element in $H^{*}\left(B G ; H_{\mathbb{Z} / p}^{*^{\prime}}\right)$ with

$$
\operatorname{Res}_{E_{2}}(x)=Q_{i_{1}} \ldots Q_{i_{n-2}}\left(u_{n}\right) .
$$

Then $w(x)=n-(n-2)=2$. However the weight of the differential $d_{r}$ of the coniveau spectral sequence is $w\left(d_{r}\right)=1-2 r$ (see the proof of Lemma 4.4). Hence $d_{r}(x)=0$ for $r \geq 2$, namely, $x$ is a permanent cycle and is an element in $H^{*}(B G ; \mathbb{Z} / p)$.

$$
\text { 6. } W_{G}(A) \cong S L_{3}(\mathbb{Z} / p)
$$

We consider the cases of $A \cong(\mathbb{Z} / p)^{3}$ and $W_{G}(A) \cong S L_{3}(\mathbb{Z} / p)$, namely, $\left(G_{2}, 2\right),\left(F_{4}, 3\right)$ and $\left(E_{8}, 5\right)$. These cases

$$
\operatorname{Inv}^{*}(G ; \mathbb{Z} / p) \cong \mathbb{Z} / p\left\{1, u_{3}\right\} .
$$

(These $u_{3}$ are called the Rost invariants.)

Let $G=G_{2}$ and $p=2$. It is well known that

$$
H^{*}(B G ; \mathbb{Z} / 2) \cong I\left(G L_{3}\right) \cong \mathbb{Z} / 2\left[w_{4}, w_{6}, w_{7}\right]
$$

where $w_{i}$ is the Stiefel-Whitney class of $G_{2} \subset S O_{7}$. We can identify

$$
w_{4}=d_{3,2}, \quad w_{6}=d_{3,1}, \quad w_{7}=d_{3,0} .
$$

On the other hand, Kameko-Mimura theorem implies

$$
\operatorname{Igr}\left(G L_{3}\right) \cong \mathbb{Z} / 2\left[c_{4}, c_{6}\right] \otimes\left(\mathbb{Z} / 2\{1\} \oplus \mathbb{Z} / 2\left[c_{7}\right] \otimes Q(2)\left\{u_{3}\right\}\right) .
$$

Also by using Lemma 5.8, we can show

$$
\operatorname{Igr}\left(G L_{3}\right) / I\left(G L_{3}\right) \cong \mathbb{Z} / 2\left[c_{4}, c_{6}\right]\left\{u_{3}\right\} .
$$

In fact (from Lemma 5.7 or from dimensional reason), we have

$$
Q_{0}\left(u_{3}\right)=w_{4}, Q_{1}\left(u_{3}\right)=w_{6}, Q_{0} Q_{1}\left(u_{3}\right)=w_{7}, Q_{2}\left(u_{3}\right)=w_{4} w_{6} .
$$

Moreover we note

$$
c_{7} u_{3}=w_{4} w_{6} w_{7}
$$

because both above elements are same after acting $Q_{i}$, e.g. $Q_{0}\left(c_{7} u_{3}\right)=$ $c_{7} w_{4}=Q_{0}\left(w_{4} w_{6} w_{7}\right)$ (see the proof of Lemma 5.7 or see [Ya2]).

Therefore Assumption (1),(2) are satisfied. Moreover from Lemma 4.4, we see $d_{2}\left(u_{3}\right)=y \neq 0$. Therefore we have the following theorem.

Theorem 6.1. There is a $Q(2)$ bidegree module epimorphism from $H^{*}\left(B G_{2} ; H_{\mathbb{Z} / 2}^{*^{\prime}}\right)$ to

$\operatorname{Igr}\left(G L_{3}\right) \oplus \mathbb{Z} / 2\left[c_{4}, c_{6}\right]\{y\} \cong \mathbb{Z} / 2\left[c_{4}, c_{6}\right] \otimes\left(\mathbb{Z} / 2\{1, y\} \oplus \mathbb{Z} / 2\left[c_{7}\right] \otimes Q(2)\left\{u_{3}\right\}\right)$. 
Remark. If there is a Gottlib transfer in the motivic theory $H^{*, *^{\prime}}(-; \mathbb{Z} / 2)$ or the sheaf theory $H^{*}\left(-; H_{\mathbb{Z} / 2}^{*}\right)$, then the above epimorphism is in fact an isomorphism.

Next we consider the odd prime cases i.e., $(G, p)=\left(F_{4}, 3\right)$ or $\left(E_{8}, 5\right)$. From Kameko-Mimura theorem, we also have

$$
\operatorname{Igr}\left(S L_{3}\right) \cong D_{3}^{\prime} \oplus S D_{3} \otimes Q(2)\left\{u_{3}\right\} .
$$

Moreover from Kameko (Lemma 5.2 in [Ka-Ya1]), it is known that

$$
\operatorname{Igr}\left(S L_{3}\right) / \operatorname{Res}_{H \mathbb{Z} / p} \cong S D_{3} /(e)\left\{u_{3}\right\}
$$

as the case $\left(G_{2}, 2\right)$. Hence Assumption (1) satisfied and Lemma 4.4 can be applied so that $d_{2}\left(u_{3}\right)=y$.

To see Assumption (2), we consider the representations. We consider the case $\left(E_{8}, 5\right)$. (The case $\left(F_{4}, 3\right)$ is similar.) It is known that there is a non trivial representation ([Ad], [Ka-Ya2])

$$
\rho: E_{8} \rightarrow S O(248) \text {. }
$$

We consider the total Chern class of the representation $\rho \mid A$ for $A \cong$ $(\mathbb{Z} / 5)^{3}$,

$$
c(\rho \mid A)=\left(1-c_{3,2}+c_{3,1}-c_{3,0}\right)^{a} \quad \text { for } a \geq 0
$$

from Lemma 5.1. Since $\rho \mid A$ is non trivial, $a \geq 1$. Moreover

$$
\left|c_{3,0}\right|=2\left(5^{3}-1\right)=248 .
$$

So $a=1$. This means that $c_{3, i}$ are represented by Chern classes. (We also note $c_{3,1}=P^{1} c_{3,2}$ for the reduced power operation $P^{1}$.) Hence $w\left(c_{3,1}\right)=w\left(c_{3,2}\right)=0$. Thus we can see Assumption (2).

Theorem 6.2. Let $(G, p)=\left(F_{4}, 3\right)$ or $\left(E_{8}, 5\right)$. Then there is an epimorphism of $Q(2)$-bidegree modules from $H^{*}\left(B G ; H_{\mathbb{Z} / p}^{*^{\prime}}\right)$ to

$$
\mathbb{Z} / p\left[c_{3,2}, c_{3,1}\right] \otimes\left(\mathbb{Z} / p\{1, y\} \oplus \mathbb{Z} / p\left[e_{3}\right] \otimes Q(2)\left\{u_{3}\right\}\right) .
$$

$$
\text { 7. } W_{G}(A) \cong\left\langle U_{4}, S L_{3}(\mathbb{Z} / p)\right\rangle
$$

We consider the cases of $A \cong(\mathbb{Z} / p)^{4}$ and

$$
W_{4}=W_{G}(A) \cong\left\langle U_{4}, S L_{3}(\mathbb{Z} / p)\right\rangle,
$$

namely, $\left(\operatorname{Spin}_{7}, 2\right),\left(E_{6}, 3\right)$. For these cases, we have the isomorphism

$$
\operatorname{Inv}^{*}(G ; \mathbb{Z} / p) \cong \mathbb{Z} / p\left\{1, u_{3}, u_{4}\right\} \text {. }
$$

We also study the case $\left(E_{7}, 3\right)$, while the above facts do not satisfied.

Let $G=\operatorname{Spin}_{7}$ and $p=2$. It is well known that

$$
H^{*}(B G ; \mathbb{Z} / 2) \cong I\left(W_{4}\right)=\mathbb{Z} / 2\left[x_{1}, \ldots, x_{4}\right]^{W_{4}}
$$




$$
\cong \mathbb{Z} / 2\left[w_{4}, w_{6}, w_{7}, w_{8}\right]
$$

where $w_{8}$ is the Stiefel-Whitney class of some spin representation. We can identify $w_{8}=\mathcal{O}_{3}^{\prime}\left(x_{4}\right)$.

\section{Lemma 7.1.}

$\operatorname{Igr}\left(W_{4}\right) \cong \operatorname{Igr}\left(G L_{3}\right) \oplus \mathbb{Z} / 2\left[c_{4}, c_{6}\right] \otimes\left(\mathbb{Z} / 2\left[c_{8}\right]\left\{c_{8}\right\} \oplus \mathbb{Z} / 2\left[c_{7}, c_{8}\right] \otimes Q(3)\left\{u_{4}\right\}\right)$.

Proof. Recall that using $\bar{Q}(2)$, we have $\left(Q_{2} Q_{1} Q_{0}\left(u_{3}\right)=c_{7}\right)$

$$
\operatorname{Igr}\left(G L_{3}\right) \cong \mathbb{Z} / 2\left[c_{4}, c_{6}, c_{7}\right] \otimes\left(\mathbb{Z} / 2\{1\} \oplus \bar{Q}(2)\left\{u_{3}\right\}\right) .
$$

By Theorem 5.4, we have

$$
\operatorname{Igr}\left(W_{4}\right) \cong \mathbb{Z} / 2\left[c_{4}, c_{6}, c_{7}, c_{8}\right] \otimes\left(\mathbb{Z} / 2\{1\} \oplus \bar{Q}(2)\left\{u_{3}\right\} \oplus Q(2)\left\{u_{4}\right\}\right)
$$

$\cong \mathbb{Z} / 2\left[c_{4}, c_{6}\right] \otimes\left(\mathbb{Z} / 2\left[c_{8}\right]\{1\} \oplus \mathbb{Z} / 2\left[c_{7}\right] \otimes Q(2)\left\{u_{3}\right\} \oplus \mathbb{Z} / 2\left[c_{7}, c_{8}\right] \otimes Q(3)\left\{u_{4}\right\}\right)$.

The last isomorphism is shown by using the following facts. From Lemma 5.6, we can see (Lemma 5.8 in [Ka-Ya2])

$$
\mathcal{O}_{3}\left(y_{4}\right) u_{3}=\left(Q_{3}+c_{3,2} Q_{2}+c_{3,1} Q_{1}+c_{3,0} Q_{0}\right)\left(u_{4}\right),
$$

namely, $Q_{3}\left(u_{4}\right)=c_{8} u_{3}+c_{4} Q_{2}\left(u_{4}\right)+c_{6} Q_{1}\left(u_{4}\right)+c_{7} Q_{0}\left(u_{4}\right)$. Hence

$$
Q(2)\left\{u_{4}\right\} \oplus Q(2)\left\{c_{8} u_{3}\right\} \cong Q(3)\left\{u_{4}\right\} .
$$

Using $Q_{0} Q_{1} Q_{2} Q_{3}\left(u_{4}\right)=c_{7} c_{8}$, we get the last isomorphism.

\section{Lemma 7.2.}

$$
\begin{gathered}
\operatorname{Igr}\left(W_{4}\right) / I\left(W_{4}\right) \cong \operatorname{Igr}\left(G L_{3}\right) / I\left(G L_{3}\right) \oplus \\
\mathbb{Z} / 2\left[c_{4}, c_{6}, c_{8}\right] \otimes\left\{1, Q_{0}, \ldots, Q_{3}\right\}\left\{u_{4}\right\} .
\end{gathered}
$$

Proof. At first, we see

$$
\begin{aligned}
& Q_{1} Q_{0}\left(u_{4}\right)=Q_{1} Q_{0}\left(u_{3} x_{4}\right)=Q_{1}\left(w_{4} x_{4}+u_{3} y_{4}\right) \\
= & w_{7} x_{4}+w_{4} y_{4}^{2}+w_{6} y_{4}=w_{8} \quad \text { in } H^{*}\left(B A ; H_{\mathbb{Z} / 2}^{*^{\prime}}\right) .
\end{aligned}
$$

(This fact also follows from $d_{4,3}=w_{8}$ and Lemma 5.7.) Similarly, we can compute the $Q_{i}$ action on $u_{4}$, which is given as $Q_{0} Q_{1}\left(u_{4}\right)=w_{8}$, $Q_{0} Q_{2}\left(u_{4}\right)=w_{4} w_{8}, Q_{1} Q_{2}\left(u_{4}\right)=w_{6} w_{8}, Q_{0} Q_{3}\left(u_{4}\right)=c_{8} w_{4}, Q_{1} Q_{3}\left(u_{4}\right)=$ $c_{8} w_{6}, Q_{2} Q_{3}\left(u_{4}\right)=c_{8} w_{4} w_{6}$.

Moreover we have

$$
c_{7} u_{4}=c_{7} u_{3} x_{4}=w_{4} w_{6} w_{7} x_{4}=w_{4} w_{6} w_{8} .
$$

Therefore $Q_{i} u_{3} \notin I\left(W_{4}\right)$ but $Q_{i} Q_{j}\left(u_{3}\right) \in I\left(W_{4}\right)$. Thus we have $\operatorname{Igr}\left(W_{4}\right) / I\left(W_{4}\right) \cong \mathbb{Z} / 2\left[c_{4}, c_{6}\right] \otimes\left(\mathbb{Z} / 2\left\{u_{3}\right\} \oplus \mathbb{Z} / 2\left[c_{8}\right]\left\{1, Q_{0}, \ldots, Q_{3}\right\}\left\{u_{4}\right\}\right)$.

Therefore Assumption (1),(2) are satisfied. Therefore we can compute ; 
Theorem 7.3. There is a $Q(2)$ bidegree module epimorphism from $H^{*}\left(\operatorname{BSpin}_{7} ; H_{\mathbb{Z} / 2}^{*^{\prime}}\right)$ to

$H^{*}\left(B G_{2} ; H_{\mathbb{Z} / 2}^{*^{\prime}}\right) \oplus \mathbb{Z} / 2\left[c_{4}, c_{6}, c_{8}\right] \otimes\left(\mathbb{Z} / 2\left\{c_{8}, y_{2}^{\prime}, Q_{0} y_{2}^{\prime}, \ldots, Q_{3} y_{2}^{\prime}\right\} \oplus \mathbb{Z} / 2\left[c_{7}\right] \otimes Q(3)\left\{u_{4}\right\}\right)$

where $Q_{0} Q_{1} Q_{2} Q_{3} u_{4}=c_{7} c_{8}$. The differentials $d_{2}\left(u_{3}\right)=y_{2}, d_{2}\left(u_{4}\right)=y_{2}^{\prime}$ in the coniveau spectral sequence.

Remark. If the epimorphism in Theorem 6.1 is an isomorphism, then that in the above theorem is also an isomorphism.

Remark. The notations in [Ya3] are given : $a^{\prime}=u_{4}$ as a virtual element and

$$
\xi_{3}=Q_{0} y_{2}^{\prime}, \xi_{4}=Q_{1} y_{2}^{\prime}, \quad \xi_{6}=Q_{2} y_{2}^{\prime}, c_{8} y_{2}=Q_{3} y_{2}^{\prime} .
$$

Next we consider the odd prime cases i.e., $(G, p)=\left(E_{6}, 3\right)$. Let us denote by $\mathcal{O}$ simply, $\mathcal{O}_{3}\left(x_{4}\right)$ so that $e_{4}=Q_{0} Q_{1} Q_{2} Q_{3}\left(u_{4}\right)=e_{3} \mathcal{O}$. Then from Kameko-Mimura lemma, we also have ([Ka-Mi],

$$
\begin{gathered}
\operatorname{Igr}\left(W_{4}\right) \cong S D /(e)[\mathcal{O}] \oplus S D_{3}[\mathcal{O}] \otimes Q(2)\left\{u_{3}, u_{4}\right\} \\
\cong S D_{3} /(e)[\mathcal{O}] \oplus S D_{3} \otimes Q(2)\left\{u_{3}\right\} \oplus S D_{3}[\mathcal{O}] \otimes Q(3)\left\{u_{4}\right\}
\end{gathered}
$$

Moreover from Kameko (Lemma 5.2 in [Ka-Ya1]), it is known that

$\operatorname{Igr}\left(W_{4}\right) / \operatorname{Res}_{H \mathbb{Z} / p} \cong S D_{3} /(e) \otimes\left(\mathbb{Z} / 3\left\{u_{3}\right\} \oplus \mathbb{Z} / 3[\mathcal{O}]\left\{u_{4}, Q_{0} u_{4}, \ldots, Q_{3} u_{4}\right\}\right)$

as the case $\left(\operatorname{Spin}_{7}, 2\right)$. Hence Assumption (1) satisfied and Lemma 4.4 can be applied so that $d_{2}\left(u_{3}\right)=y$.

To see Assumption (2), we consider the representations. It is known that there is a non trivial representation $E_{6} \rightarrow S O(26)$. Hence we know that $c_{3, i}$ is represented by Chern classes by the arguments similar to the case $\left(F_{4}, 3\right)$. As for the element $\mathcal{O}$, we consider the restriction

$$
\left\langle a_{4}\right\rangle \subset A \subset E_{6} \stackrel{\rho}{\rightarrow} S O(26) .
$$

Here $a_{4} \in A$ is the dual of $x_{4} \in \operatorname{Hom}(A ; \mathbb{Z} / 3)$. We see $\mathcal{O} \mid\left\langle a_{4}\right\rangle \neq 0$ but $S D_{3} \mid\left\langle a_{4}\right\rangle=0$. Hence the fact

$$
c_{26}\left(\rho \mid\left\langle a_{4}\right\rangle\right)=y_{4}^{3^{3}-1} \neq 0
$$

implies $\mathcal{O}$ (modulo elements in $S D_{3}$ ) can be represented by a Chern class. Hence Assumption (2) is also satisfied.

Theorem 7.4. There is an epimorphism of $Q(3)$-bimodules from $H^{*}\left(B E_{6} ; H_{\mathbb{Z} / 3}^{*^{\prime}}\right)$ to

$$
\begin{gathered}
H^{*}\left(B F_{4} ; H_{\mathbb{Z} / 3}^{*^{\prime}}\right) \oplus \mathbb{Z} / 3\left[c_{3,2}, c_{3,1}, \mathcal{O}\right] \otimes\left(\mathbb{Z} / 3\left\{\mathcal{O}, y_{2}^{\prime}, Q_{0} y_{2}^{\prime}, \ldots, Q_{3} y_{2}^{\prime}\right\}\right. \\
\left.\oplus \mathbb{Z} / 2\left[e_{3}\right] \otimes Q(3)\left\{u_{4}\right\}\right) .
\end{gathered}
$$


At last of this section, we consider the case $\left(E_{7}, 3\right)$. This case

$$
W_{G}(A)=W_{4}^{\prime}=\left\langle W_{4}, \operatorname{diag}(1,1,1,-1)\right\rangle \subset G L_{4}(\mathbb{Z} / 3) .
$$

The invariant is also computed by Kameko-Mimura

$$
\operatorname{Igr}\left(W_{4}^{\prime}\right) \cong S D /(e)\left[\mathcal{O}^{2}\right] \oplus S D_{3}\left[\mathcal{O}^{2}\right] \otimes Q(2)\left\{u_{3}, \mathcal{O} u_{4}\right\}
$$

Moreover from Kameko (page 2279 in [Ka-Ya1]), it is known that

$$
\operatorname{Igr}\left(W_{4}^{\prime}\right) / \operatorname{Res}_{H \mathbb{Z} / 3} \cong S D_{3} /(e)\left[\mathcal{O}^{2}\right] \otimes\left(\mathbb{Z} / 3\left\{u_{3}\right\} \oplus Q(2)\left\{\mathcal{O} u_{4}\right\}\right) .
$$

It is also known $\operatorname{Inv}^{*}\left(E_{7} ; \mathbb{Z} / 3\right) \cong \mathbb{Z} / 3\left\{1, u_{3}\right\}$ and hence

$$
\operatorname{Inv}^{*}\left(E_{7} ; \mathbb{Z} / 3\right) \cong \operatorname{Inv}^{*}(A ; \mathbb{Z} / 3)^{W_{4}^{\prime}}
$$

from the above result.

There is the natural representation $\rho: E_{7} \rightarrow S O(52)$. Hence we see that $\mathcal{O}^{2}$ can be represented by a Chern class. So Assumption (2) is satisfied. However Assumption (1) is not.

Theorem 7.5. The following restriction map

$$
\operatorname{Res}_{E_{2}}: H^{*}\left(B E_{7}, H_{\mathbb{Z} / 3}^{*^{\prime}}\right) \rightarrow H^{*}\left(B A, H_{\mathbb{Z} / 3}^{*^{\prime}}\right)^{W_{4}^{\prime}}
$$

is not an epimorphism.

Proof. Recall arguments in the proof of Lemma 5.9. Suppose $\mathcal{O} u_{4} \in$ $\operatorname{Res}_{E_{2}}$ and $\operatorname{Res}(x)=\mathcal{O} u_{4}$ for $x \in H^{*}\left(B E_{7} ; H_{\mathbb{Z} / 3}^{*^{\prime}}\right)$.

Of course $w(x)=w\left(\mathcal{O} u_{4}\right)=4$. The weight $Q_{0} Q_{1}(x)=2$. Recall $w\left(d_{r}\right)=1-2 r$ and

$$
d_{r}\left(Q_{0} Q_{1}(x)\right)=0 \quad \text { for } r \geq 2 .
$$

Hence $Q_{0} Q_{1}(x) \in H^{*}\left(B E_{3} ; \mathbb{Z} / 3\right)$ from the coniveau spectral sequence. So $Q_{0} Q_{1}\left(\mathcal{O} u_{4}\right) \in \operatorname{Res}_{H \mathbb{Z} / 3}$, which contradicts to the result above

$$
\operatorname{Igr}\left(W_{4}^{\prime}\right) / \operatorname{Res}_{H \mathbb{Z} / 3} \supset Q(2)\left\{\mathcal{O} u_{4}\right\} .
$$

\section{8. $\operatorname{Spin}_{9}$ FOR $p=2$}

In this section, we consider the groups $\operatorname{Spin}_{8}, \operatorname{Spin}_{9}, F_{4}, E_{6}$ for $p=2$. At first we consider the case $G=S_{p i n}$. Then the maximal elementary abelian 2-group is $r_{a n k}=5$, and the Weyl group is

$$
W_{G}(A)=W_{5} \cong\left\langle U_{5}, S L_{3}\right\rangle \subset S L_{5}(\mathbb{Z} / 2) .
$$

The cohomology is known that

$$
H^{*}(B G ; \mathbb{Z} / 2) \cong H^{*}(B A ; \mathbb{Z} / 2)^{W_{G}(A)} \cong \mathbb{Z} / 2\left[w_{4}, w_{6}, w_{7}, w_{8}, w_{16}\right]
$$


where $w_{16}$ is the Stiefel-Whitney class of some spin representation. We can also identify

$$
w_{16}=\mathcal{O}_{4}^{\prime}\left(x_{5}\right)=x_{5}^{16}+d_{3} x_{5}^{8}+d_{2} x_{5}^{4}+d_{1} x_{5}^{2}+d_{0} x_{5}
$$

where $d_{3}=w_{8}, d_{2}=w_{8} w_{4}, d_{1}=w_{8} w_{6}, d_{0}=w_{8} w_{7}$ (see the proof of Lemma 7.2 or p1051 in [Sc-Ya]). As the case $(*)$ in $\S 5$, we know $([\mathrm{Sc}-\mathrm{Ya}])$

$$
Q_{3} w_{16}=d_{0} w_{16}, \quad Q_{4}\left(d_{0} w_{16}\right)=d_{0}^{2} w_{16}^{2} .
$$

We can prove from Kameko-Mimura theorem

\section{Lemma 8.1.}

$$
\begin{gathered}
\operatorname{Igr}\left(W_{5}\right) \cong \operatorname{Igr}\left(W_{4}\right) \oplus \mathbb{Z} / 2\left[c_{4}, c_{6}, c_{16}\right] \otimes\left(\mathbb{Z} / 2\left[c_{8}\right]\left\{c_{16}\right\} \oplus\right. \\
\left.\mathbb{Z} / 2\left[c_{7}\right] \otimes Q(2)\left\{c_{16} u_{3}\right\} \oplus \mathbb{Z} / 2\left[c_{7}, c_{8}\right] \otimes Q(4)\left\{u_{5}\right\}\right)
\end{gathered}
$$

Proof. Recall that

$$
\operatorname{Igr}\left(W_{4}\right) \cong \mathbb{Z} / 2\left[c_{4}, c_{6}, c_{7}, c_{8}\right] \otimes\left(\mathbb{Z} / 2\{1\} \oplus \bar{Q}(2)\left\{u_{3}\right\} \oplus Q(2)\left\{u_{3}\right\}\right) .
$$

From Kameko-Mimura theorem (Theorem 5.4) we see that

$$
\operatorname{Igr}\left(W_{5}\right) \cong \mathbb{Z} / 2\left[c_{4}, c_{6}, c_{7}, c_{8}, c_{16}\right] \otimes\left(\mathbb{Z} / 2\{1\} \oplus \bar{Q}(2)\left\{u_{3}\right\} \oplus Q(2)\left\{u_{4}\right\} \oplus Q(3)\left\{u_{5}\right\}\right) .
$$

Using $Q_{2} Q_{1} Q_{0}\left(u_{3}\right)=c_{7}$ and $Q_{3}\left(u_{4}\right)=c_{8} u_{3}+\ldots$ as the case for $\operatorname{Spin}_{7}$, we have the isomorphism

$Q(2)\left\{u_{3}\right\} \cong \mathbb{Z} / 2\left\{c_{7}\right\} \oplus \bar{Q}(2)\left\{u_{3}\right\}, \quad Q(3)\left\{u_{4}\right\} \cong Q(2)\left\{c_{8} u_{3}\right\} \oplus Q(2)\left\{u_{4}\right\}$.

Hence $\operatorname{Igr}\left(W_{5}\right)$ is isomorphic to

$$
\begin{aligned}
& \mathbb{Z} / 2\left[c_{4}, c_{6}, c_{16}\right] \otimes\left(\mathbb{Z} / 2\left[c_{8}\right] \oplus \mathbb{Z} / 2\left[c_{7}\right] \otimes Q(2)\left\{u_{3}\right\} \oplus\right. \\
& \left.\mathbb{Z} / 2\left[c_{7}, c_{8}\right] \otimes Q(3)\left\{u_{4}\right\} \oplus \mathbb{Z} / 2\left[c_{7}, c_{8}\right] \otimes Q(3)\left\{u_{5}\right\}\right)
\end{aligned}
$$

Using the fact $Q_{4}\left(u_{5}\right)=c_{16} u_{4}+\ldots$ from Lemma 5.6, we have the isomorphism

$$
Q(4)\left\{u_{5}\right\} \cong Q(3)\left\{c_{16} u_{4}\right\} \oplus Q(3)\left\{u_{5}\right\} .
$$

This induces the isomorphism

$$
\begin{gathered}
\operatorname{Igr}\left(W_{5}\right) \cong \mathbb{Z} / 2\left[c_{4}, c_{6}\right] \otimes\left(\mathbb{Z} / 2\left[c_{8}, c_{16}\right] \oplus \mathbb{Z} / 2\left[c_{7}, c_{16}\right] \otimes Q(2)\left\{u_{3}\right\}\right. \\
\left.\oplus \mathbb{Z} / 2\left[c_{7}, c_{8}\right] \otimes Q(3)\left\{u_{4}\right\} \oplus \mathbb{Z} / 2\left[c_{7}, c_{8}, c_{16}\right] \otimes Q(4)\left\{u_{5}\right\}\right) .
\end{gathered}
$$

Hence we have the desired isomorphism.

The cohomological invariant is known

$$
\operatorname{Inv}^{*}\left(\operatorname{Spin}_{9}, \mathbb{Z} / 2\right) \cong \mathbb{Z} / 2\left\{1, u_{3}, u_{4}, u_{5}\right\} .
$$

Hence Assumption (1),(2) are also satisfied for $\left(\operatorname{Spin}_{9}, 2\right)$ (from the last isomorphism in the above proof). 


\section{Lemma 8.2.}

$$
\begin{gathered}
\operatorname{Igr}\left(W_{5}\right) / I\left(W_{5}\right) \cong \operatorname{Igr}\left(W_{4}\right) /\left(W_{4}\right) \oplus \mathbb{Z} / 2\left[c_{4}, c_{6}, c_{16}\right] \otimes\left(\mathbb{Z} / 2\left\{c_{16} u_{3}\right\} \oplus\right. \\
\oplus \mathbb{Z} / 2\left[c_{8}\right] \otimes\left(\left(Q(4) /\left(Q(4)^{+}\right)^{3}\left\{u_{5}\right\} \oplus \mathbb{Z} / 2\left\{c_{7} u_{5}\right\}\right)\right) .
\end{gathered}
$$

Proof. Since

$$
Q_{0}\left(u_{5}\right)=Q_{0}\left(u_{4} x_{5}\right)=Q_{0}\left(u_{4}\right) x_{5}+u_{4} y_{5},
$$

we can compute

$$
\begin{aligned}
Q_{2} Q_{1} Q_{0}\left(u_{5}\right) & =Q_{2} Q_{1} Q_{0}\left(u_{4}\right) x_{5}+Q_{1} Q_{0}\left(u_{4}\right) y_{5}^{4}+Q_{2} Q_{0}\left(u_{4}\right) y_{5}^{2}+Q_{2} Q_{1}\left(u_{4}\right) y_{5} \\
& =w_{7} w_{8} x_{5}+w_{8} y_{5}^{4}+w_{4} w_{8} y_{5}^{2}+w_{6} w_{8} y_{5}=w_{16} .
\end{aligned}
$$

(This fact also follows from $d_{5,4}=w_{16}$.) Let us write $Q_{i_{1}, \ldots, i_{j}}\left(u_{5}\right)=$ $Q_{i_{1}, \ldots, i_{j}}$ simply. Similarly we can compute

$$
\begin{gathered}
Q_{012}=w_{16}, \quad Q_{013}=w_{16} w_{8}, \quad Q_{023}=w_{16} w_{4} w_{8}, \quad Q_{123}=w_{16} w_{6} w_{8}, \\
Q_{014}=c_{16} w_{8}, \quad Q_{024}=c_{16} w_{8} w_{4}, \quad Q_{034}=c_{16} c_{8} w_{4}, \\
Q_{124}=c_{16} w_{6} w_{8}, \quad Q_{134}=c_{16} c_{8} w_{6}, \quad Q_{234}=c_{16} c_{8} w_{4} w_{6} .
\end{gathered}
$$

We can compute

$$
\begin{aligned}
& Q_{0}\left(c_{7} u_{5}\right)=Q_{0}\left(c_{7} u_{4} x_{5}\right)=Q_{0}\left(w_{4} w_{6} w_{8} x_{5}\right) \\
& =w_{4} w_{7} w_{8} x_{5}+w_{4} w_{6} w_{8} y_{5}+\ldots=w_{4} w_{16} .
\end{aligned}
$$

Let us write $Q_{i_{1}, \ldots, i_{j}}\left(c_{7} u_{5}\right)=Q_{i_{1}, \ldots, i_{j}}^{\prime}$ simply. Then we can compute

$$
Q_{0}^{\prime}=w_{16} w_{4}, \quad Q_{1}^{\prime}=w_{16} x_{6}, \quad Q_{2}^{\prime}=w_{16} w_{4} w_{6}, \quad Q_{3}^{\prime}=w_{16} w_{4} w_{6} w_{8},
$$

$Q_{4}^{\prime}=c_{16} w_{4} w_{6} w_{8}, \quad Q_{01}^{\prime}=w_{16} w_{7}, \quad Q_{12}^{\prime}=w_{16} w_{6} w_{7}, \quad Q_{04}^{\prime}=c_{16} w_{7} w_{4} w_{8}$.

Moreover

$$
c_{7}^{2} u_{5}=c_{7} w_{4} w_{6} w_{8} x_{5}=w_{4} w_{6} w_{7} w_{16} .
$$

There appear all generators of the $\mathbb{Z} / 2\left[c_{4}, c_{6}, c_{8}, c_{16}\right]$-module with modulo $\operatorname{Ideal}\left(c_{7}, w_{7}\right)$. Thus we can see

$$
\begin{gathered}
\operatorname{Igr}\left(W_{5}\right) / I\left(W_{5}\right) \cong \mathbb{Z} / 2\left[c_{4}, c_{6}\right] \otimes\left(\mathbb{Z} / 2\left[c_{16}\right]\left\{u_{3}\right\} \oplus \mathbb{Z} / 2\left[c_{8}\right]\left(Q(3) /\left(Q(3)^{+}\right)^{2}\left\{u_{4}\right\}\right.\right. \\
\oplus \mathbb{Z} / 2\left[c_{8}, c_{16}\right] \otimes\left(\left(Q(4) /\left(Q(4)^{+}\right)^{3}\left\{u_{5}\right\} \oplus \mathbb{Z} / 2\left\{c_{7} u_{5}\right\}\right)\right) .
\end{gathered}
$$

Theorem 8.3. There is an epimorphism of $Q(4)$-modules from $H^{*}\left(\right.$ BSpin $\left._{9} ; H_{\mathbb{Z} / 2}^{*^{\prime}}\right)$ to $H^{*}\left(\operatorname{BSpin}_{7} ; H_{\mathbb{Z} / 3}^{*^{\prime}}\right) \oplus \mathbb{Z} / p\left[c_{4}, c_{6}, c_{16}\right] \otimes\left(\mathbb{Z} / 2\left[c_{7}\right] \otimes Q(2)\left\{c_{16} u_{3}\right\} \oplus \mathbb{Z} / 2\left\{c_{16} y\right\}\right.$

$$
\oplus \mathbb{Z} / 2\left[c_{8}, c_{7}\right] \otimes Q(4)\left\{u_{5}\right\} \oplus \mathbb{Z} / 2\left[c_{8}\right] \otimes\left(Q(4) /\left(Q(4)^{+}\right)^{3}\left\{y^{\prime \prime}\right\}\right)
$$

where $d_{2} u_{3}=y$ and $d_{2}\left(u_{5}\right)=y^{\prime \prime}$. 
Remark. However note that we can not see $d_{2}\left(c_{7} u_{5}\right) \neq 0$ or not.

From Lemma 4.4 , we know $d_{2}\left(u_{5}\right)=y^{\prime \prime} \neq 0$ and we get many Griffith elements

$$
Q_{i} Q_{j}\left(y^{\prime \prime}\right) \quad \text { for } 0 \leq i<j \leq 4 .
$$

We study the image of the cycle map $\tilde{c l}$ to $B P^{*}\left(B \operatorname{Spin}_{9}\right) \otimes_{B P^{*}} \mathbb{Z} / 2$ in the last section (indeed $\tilde{c l}\left(Q_{i} Q_{j}\left(y^{\prime \prime}\right) \neq 0\right)$.

Next we consider the case $\left(\operatorname{Spin}_{8}, 2\right)$. The Weyl group is

$$
W_{4} \subset W_{G}(A)=W_{5}^{\prime \prime}=\left\{\left(w_{i j}\right) \in G L_{5}(\mathbb{Z} / 2) \mid w_{5,4}=0\right\} \subset W_{5} .
$$

We can compute

$$
\begin{gathered}
\operatorname{Igr}\left(W_{5}^{\prime \prime}\right) \cong \operatorname{Igr}\left(W_{4}\right) \oplus \mathbb{Z} / 2\left[c_{4}, c_{6}, c_{8}^{\prime}, c_{8}\right] \otimes \\
\left(\mathbb{Z} / 2\left\{c_{8}^{\prime}\right\} \oplus \mathbb{Z} / 3\left[c_{7}\right] \otimes Q(3)\left\{u_{4}^{\prime}\right\} \otimes \mathbb{Z} / 2\left[c_{7}\right] \otimes Q(4)\left\{u_{5}\right\}\right) .
\end{gathered}
$$

Hence Assumption (1) (with some modification for $u_{4}^{\prime}$ ) and (2) are satisfied.

We consider the case $\left(F_{4}, 2\right)$ also . This case $A \cong(\mathbb{Z} / 2)^{5}$ but

$$
W_{G}(A)=W_{5}^{\prime}=\left\langle U_{5}, G L_{3}(\mathbb{Z} / 2) \oplus G L_{2}(\mathbb{Z} / 2)\right\rangle \subset G L_{5}(\mathbb{Z} / 2) .
$$

The cohomology is given by

$$
H^{*}(B G ; \mathbb{Z} / 2) \cong H^{*}(B A ; \mathbb{Z} / 2)^{W_{G}(A)} \cong \mathbb{Z} / 2\left[w_{4}, w_{6}, w_{7}, x_{16}, x_{24}\right]
$$

where $x_{16}=w_{8}^{2}+w_{16}$ and $x_{24}=w_{8} w_{16}$. We consider the representation

$$
\left\langle a_{4}, a_{5}\right\rangle \subset A \subset F_{4} \stackrel{\rho}{\rightarrow} S O(26)
$$

where $a_{4}, a_{5}$ are dual of $x_{4}, x_{5}$. Then the total Chern class is

$$
c\left(\rho \mid\left\langle a_{4}, a_{5}\right\rangle\right)=\left(1+c_{2,1}+c_{2,0}\right)^{a}
$$

from Lemma 5.1. By dimensional reason, $a \leq 8$. So we see $x_{16}^{2}$ and $x_{24}^{2}$ are represented by Chern classes. Thus we can write

$$
\begin{gathered}
\operatorname{Igr}\left(W_{5}^{\prime}\right) \cong \mathbb{Z} / 2\left[c_{4}, c_{6}\right] \otimes\left(\mathbb{Z} / 2\left[c_{16}, c_{24}\right] \oplus \mathbb{Z} / 2\left[c_{7}, c_{24}\right] \otimes Q(2)\left\{u_{3}\right\}\right. \\
\left.\oplus \mathbb{Z} / 2\left[c_{7}, c_{16}, c_{24}\right] \otimes Q(4)\left\{u_{5}\right\}\right) .
\end{gathered}
$$

Hence Assumption (1),(2) are also satisfied. So $R e s_{E_{2}}$ is an epimorphism for $\left(F_{4}, 2\right)$.

At last of examples, we consider the case $\left(E_{6}, 2\right)$. This case $W_{G}(A) \cong$ $W_{5}^{\prime}$ same as the case $F_{4}$. But it is known ([Ga-Me-Se]) that

$$
\operatorname{Inv}^{*}\left(E_{6}, \mathbb{Z} / 2\right) \cong \mathbb{Z} / 2\left\{1, u_{3}\right\} \text {. }
$$

Therefore we see

Lemma 8.4. When $G=E_{6}$ and $p=2$, the restriction map

$$
\operatorname{Res}_{E_{2}}: H^{*}\left(B G ; H_{\mathbb{Z} / 2}^{*^{\prime}}\right) \rightarrow H^{*}\left(B A ; H_{\mathbb{Z} / 2}^{*^{\prime}}\right)^{W_{G}(A)}
$$

is not an epimorphism. 
The above fact also proved by using the cohomology $H^{*}\left(B E_{6} ; \mathbb{Z} / 2\right)$ and Lemma 5.9 as follows.

Theorem 8.5. The restriction maps

$$
\operatorname{Res}_{\text {Inv }}: \operatorname{Inv}^{*}(G ; \mathbb{Z} / 2) \rightarrow \operatorname{Inv}^{*}\left(A_{5} ; \mathbb{Z} / 2\right)^{W_{G}\left(A_{5}\right)}
$$

are epimorphisms for $G=E_{6}$ and $G=\operatorname{Spin}_{n}, n \geq 10$.

Proof. Of course there is the embedding $i: \operatorname{Spin}_{9} \subset \operatorname{Spin}_{10}$. From Kono-Mimura [Ko-Mi], we know

$$
H^{*}\left(\operatorname{BSpin}_{10} ; \mathbb{Z} / 2\right) \cong H^{*}\left(\operatorname{BSpin}_{7} ; \mathbb{Z} / 2\right) \otimes \mathbb{Z} / 2\left[x_{10}, x_{32}\right] /\left(w_{7} x_{10}\right) .
$$

Hence $H^{*}\left(B E_{6} ; \mathbb{Z} / 2\right)$ does not contain an element $x$ with $i^{*}(x)=w_{16}$ for $w_{16}=d_{5,4}=Q_{0} Q_{1} Q_{2}\left(u_{5}\right)$. From Lemma 5.9, we see that $\operatorname{Res}_{I n v}$ is not an epimorphism.

There is the embedding $F_{4} \subset E_{6}$. From also Kono-Mimura [Ko-Mi], we know

$H^{*}\left(B E_{6} ; \mathbb{Z} / 2\right) \cong H^{*}\left(\right.$ BSpin $\left._{7} ; \mathbb{Z} / 2\right) \otimes \mathbb{Z} / 2\left[x_{10}, x_{18}, x_{32}, x_{48}\right] /($ relations $)$.

Hence $\operatorname{Res}_{\text {Inv }}$ is not epic from the lack of element $x_{16}$.

\section{BP-THEORY AND GRIFFITH ELEMENTS}

In this section, we recall the results in $\S 5$ in [Ya1] and consider the relation between $B P$-theory and results in the preceding sections. We always assume $k=\mathbb{C}$ in this section. Let $B P^{*}(-)$ be the Brown-Peterson theory with the coefficient $\operatorname{ring} B P^{*}=\mathbb{Z}_{(p)}\left[v_{1}, \ldots\right],\left|v_{i}\right|=-2\left(p^{i}-1\right)$. The Thom map induces $\rho: B P^{*}(X) \otimes_{B P^{*}} \mathbb{Z}_{(p)} \rightarrow H^{*}\left(X ; \mathbb{Z}_{(p)}\right)$. Totaro constructs [To1] the map

$$
\tilde{c l}: C H^{*}(X)_{(p)} \rightarrow B P^{*}(X) \otimes_{B P^{*}} \mathbb{Z}_{(p)}
$$

such that the composition $\rho \cdot \tilde{c l}$ is the usual cycle map $c l=t_{\mathbb{C}}$ which is also the realization map. Totaro conjectured that this map is isomorphic for $X=B G$.

Let us write by

$$
P(n)^{*}=B P^{*} /\left(p, v_{1}, \ldots, v_{n-1}\right),
$$

e.g., $P(0)^{*}=B P^{*}, P(1)^{*}=B P^{*} / p$ and $P(\infty)^{*}=\mathbb{Z} / p$.

Many cases of $X([\mathrm{Te}-\mathrm{Ya} 2]$, [Ko-Ya] $), B P^{*}(X)$ are computed by the Atiyah-Hirzebruch spectral sequences

$$
E_{2}^{*, *^{\prime}}=H^{*}(X) \otimes B P^{*^{\prime}} \Longrightarrow B P^{*}(X) \text {. }
$$

It is known that $d_{2 p^{i}-1}(x)=v_{i} \otimes Q_{i}(x) \bmod \left(M_{i}\right)$ where $M_{i}$ is the ideal of $E_{2 p^{i}-1}^{*, *^{\prime}}$ generated by elements in $\left(p, \ldots, v_{i-1}\right) E_{2}^{*, *^{\prime}}$. 
We assume that $H^{*}(X)$ has no higher $p$-torsion and all non zero differentials are of form

$$
d_{2 p^{i}-1}(x)=v_{i} \otimes Q_{i}(x) \bmod \left(M_{i}\right)
$$

Let us write

$$
\text { (9.2) } \operatorname{gr} B P^{*}(X) \cong E_{\infty}^{*, *} \cong A \oplus B
$$

where $A$ (resp. $B$ ) is a $B P^{*}$-module generated by elements in $H^{*}(X) / p$ (resp. $p H^{*}(X) \oplus E_{\infty}^{* \text { minus }}$ ) so that $B \subset \operatorname{Ker}\left(\rho_{p}\right)$. Then we can write

$$
A \cong \oplus_{n=-1} P(n+1)^{*} \tilde{G}_{n}
$$

by the prime invariant ideal theorem of Landweber; if $P(n)^{*} /(a)$ is a $B P^{*}(B P)$-module, then $a=v_{n}^{s}$ for some $s \geq 1$.

Lemma 9.1. (Lemma 5.1 in [Ya1]) Let $H^{*}(X)_{(p)}$ has no higher $p$ torsion. Suppose (9.1) and $A=\oplus_{n=-1} P(n+1)^{*} \tilde{G}_{n}$ in (9.2). Then there is a injection of $Q(\infty)$-modules

$$
H^{*}(X ; \mathbb{Z} / p) \hookrightarrow \oplus_{n=-1} Q(n) G_{n} \text { with } Q_{0} \ldots Q_{n} G_{n}=\tilde{G}_{n}
$$

It is proved ([Ko-Ya],[Ka-Ya],[Ya1]) that all $X=B G$ in Theorem 1.2 satisfy the assumption in the above lemma. Hence

$$
H^{*}(B G ; \mathbb{Z} / p) \hookrightarrow \oplus_{n=-1}^{\infty} Q(n) G_{n} .
$$

Moreover when $n \neq-1$, we still know

$$
w\left(G_{n}\right)=n \text {. }
$$

Indeed if $n \geq 0$, then $G_{n}=G_{n}^{\prime}\left\{u_{n}\right\}$ where $G_{n}^{\prime}$ is represented by elements in $C H^{*}(B G) / p$ (see Assumption (1),(2)). From Theorem 1.2, we also know

Corollary 9.2. Let $G$ be a group in Theorem 1.2. Then there is a bidegree $Q(n)$-module injection

$$
\oplus_{i \geq 0} Q(n) G_{n} \subset H^{*}\left(B G ; H_{\mathbb{Z} / p}^{*^{\prime}}\right) .
$$

Lemma 9.3. (Lemma 5.2 in $[Y a])$ Let $H^{*}(X)_{(p)}$ has no higher $p$ torsion.

(1) If (9.1) is satisfied and in (9.2),

$$
A \cong \oplus_{n=-1} P(n+1)^{*} \tilde{G}_{n} \text { and } B \cong \oplus_{s=0} B P^{*}\left\{p, v_{1}, . ., v_{s}\right\} \tilde{K}_{s},
$$

then we have the isomorphism

$$
H^{*}(X ; \mathbb{Z} / p) \cong\left(\oplus_{n=-1} Q(n) G_{n}\right)-\left(\oplus_{s} \bar{Q}(s) K_{s}\right)
$$

with $Q_{0} \ldots Q_{n} G_{n}=\tilde{G}_{n}$, and $Q_{1} \ldots Q_{s} K_{s}=\tilde{K}_{s}$.

(2) If $Q_{0} \ldots Q_{n} G_{n} \in \operatorname{Im}(\rho)$ and $\left|Q_{1} \ldots Q_{s} K_{s}\right|=$ even, then the converse also holds. 
Corollary 9.4. Let $G$ be a group in Theorem 1.2 so that Assumption (1),(2) are satisfied. Then there are epimorphisms from $\operatorname{gr} B P^{*}(B G) \cong$ $E_{\infty}^{*, *^{\prime}}$ to

$$
\oplus_{s} P\left(i_{s}\right)^{*}\left[f_{s 1}, \ldots, f_{s k_{s}}\right]\left\{\tilde{u}_{i_{s}}\right\} \oplus \oplus_{t} B P^{*}\left(p, \ldots, v_{t}\right)\left\{\tilde{K}_{t}\right\}
$$

and from $B P^{*}(B G) \otimes_{B P^{*}} \mathbb{Z} / p$ to

$$
\oplus_{s} \mathbb{Z} / p\left[f_{s 1}, \ldots, f_{s k_{s}}\right]\left\{\tilde{u}_{i_{s}}\right\} \oplus \oplus_{t} \mathbb{Z} / p\left\{p, \ldots, v_{t}\right\}\left\{\tilde{K}_{t}\right\}
$$

where $\tilde{u}_{i_{s}}=Q_{i_{s}-1} \ldots Q_{0}\left(u_{i_{s}}\right)$ and $\operatorname{Igr}(W) / \operatorname{Res}_{H \mathbb{Z} / p} \cong \oplus_{t} \bar{Q}(t) K_{t}$.

We give examples. At first we recall the Atiyah-Hirzebruch spectral sequence for $B G_{2}$ in [Ko-Ya]. Since $H^{*}(B G)$ has no higher torsin, we have

$$
H^{*}\left(B G_{2}\right)_{(2)} \cong \mathbb{Z}_{(2)}\left[w_{4}, c_{6}\right] \otimes\left(\mathbb{Z}_{(2)}\{1\} \oplus \mathbb{Z} / 2\left[w_{7}\right]\left\{w_{7}\right\}\right) .
$$

Let us write $B_{i_{1}, \ldots, i_{j}}=\mathbb{Z}_{(2)}\left[c_{i_{1}}, \ldots, c_{i_{j}}\right]$, e.g., $B_{4,6}=\mathbb{Z}_{(2)}\left[c_{4}, c_{6}\right]$.

Since $Q_{1}\left(w_{4}\right)=w_{7}$, we have $d_{3}\left(w_{4}\right)=v_{1} \otimes w_{7}$. Hence the $E_{4}$-term of the spectral sequence is

$$
E\left(G_{2}\right)_{4}^{*, *} \cong B_{4,6} \otimes\left(B P^{*}\left\{1,2 w_{4}\right\} \oplus P(2)^{*}\left[c_{7}\right]\left\{c_{7}, w_{7}\right\}\right) .
$$

Next differential is $d_{7}\left(w_{7}\right)=v_{2} \otimes Q_{2}\left(w_{7}\right)=v_{2} c_{7}$ and

$$
E\left(G_{2}\right)_{8}^{*, *} \cong B_{4,6} \otimes\left(B P^{*}\left\{1,2 w_{4}\right\} \oplus P(3)^{*}\left[c_{7}\right]\left\{c_{7}\right\}\right) .
$$

which is isomorphic to $E\left(G_{2}\right)_{\infty}^{*, *}$. In particular

$$
B P^{*}(B G) \otimes_{B P^{*}} \mathbb{Z} / 2 \cong \mathbb{Z} / 2\left[c_{4}, c_{6}\right] \otimes\left(\mathbb{Z} / 2\left\{1,2 w_{4}\right\} \oplus \mathbb{Z} / 2\left[c_{7}\right]\left\{c_{7}\right\}\right) .
$$

This result is also immediate from Corollary 9.4 and Theorem 6.1, in fact, we have the epimorphism

$$
H^{*}\left(B G_{2} ; H_{\mathbb{Z} / 2}^{*^{\prime}}\right) \rightarrow \mathbb{Z} / 2\left[c_{4}, c_{6}\right] \otimes\left(\mathbb{Z} / 2\{1, y\} \oplus \mathbb{Z} / 2\left[c_{7}\right] \otimes Q(2)\left\{u_{3}\right\}\right) .
$$

Here $\tilde{u}_{3}=Q_{0} Q_{1} Q_{2}\left(u_{3}\right)=c_{7}$ and $d_{2}\left(u_{3}\right)=y$ in the coniveau spectral sequence, and we have

$$
\bar{Q}(0) K_{0}=K_{0}=\mathbb{Z} / 2\left[c_{4}, c_{6}\right]\{u\} .
$$

Hence the cycle map $\tilde{c l}$ is epimorphism and $\tilde{c l}(y)=\left\{2 w_{4}\right\}$ (which is represented by a Chern class $c_{2}$ ). Moreover we know [Ya2] that this $\tilde{c l}$ is a really isomorphism.

Next consider the case $\operatorname{Spin}_{7}$. From [Ko-Ya], we can compute

$$
\begin{gathered}
E\left(\operatorname{Spin}_{7}\right)_{16}^{*, *} \cong B_{4,6} \otimes P(3)^{*}\left[c_{7}\right]\left\{c_{7}\right\} \oplus \\
B_{4,6,8} \otimes\left(B P^{*}\left\{1,2 w_{4}, 2 w_{4} w_{8}, 2 w_{8}, v_{1} w_{8}\right\} \oplus P(4)^{*}\left[c_{7}\right]\left\{c_{7} c_{8}\right\}\right) .
\end{gathered}
$$

This term is also the infinity term. Hence we have

$$
B P^{*}(B G) \otimes_{B P^{*}} \mathbb{Z} / 2 \cong B P^{*}\left(B G_{2}\right) \otimes_{B P^{*}} \mathbb{Z} / 2 \oplus
$$


$\mathbb{Z} / 2\left[c_{4}, c_{6}, c_{8}\right] \otimes\left(\mathbb{Z} / 2\left\{c_{8}, 2 c_{8} w_{4}, 2 w_{4} w_{8}, 2 w_{8}, v_{1} w_{8}\right\} \oplus \mathbb{Z} / 2\left[c_{7}\right]\left\{c_{7} c_{8}\right\}\right)$

This result is also get from Corollary 9.4 and Theorem 7.3, indeed, we have the epimorphsm

$$
H^{*}\left(\operatorname{BSpin}_{7} ; H_{\mathbb{Z} / 2}^{*^{\prime}}\right) \rightarrow H^{*}\left(B G_{2} ; H_{\mathbb{Z} / 2}^{*^{\prime}}\right) \oplus
$$

$\mathbb{Z} / 2\left[c_{4}, c_{6}, c_{8}\right] \otimes\left(\mathbb{Z} / 2\left\{c_{8}, y_{2}^{\prime}, Q_{0} y_{2}^{\prime}, \ldots, Q_{3} y_{2}^{\prime}\right\} \oplus \mathbb{Z} / 2\left[c_{7}\right] \otimes Q(3)\left\{u_{4}\right\}\right)$

This case $d_{2}\left(u_{3}\right)=y, d_{2}\left(u_{4}\right)=y^{\prime}$ in the coniveau spectral sequence.

Recall

$$
\begin{gathered}
\oplus_{s} \bar{Q}(s) K_{s} \cong \operatorname{Igr}\left(W_{4}\right) / I\left(W_{4}\right) \cong I\left(G L_{3}\right) / I\left(G L_{3}\right) \oplus \\
\mathbb{Z} / 2\left[c_{4}, c_{6}, c_{8}\right]\left\{1, Q_{0}, \ldots, Q_{3}\right\}\left\{u_{4}\right\} .
\end{gathered}
$$

We can take

$$
\begin{gathered}
\bar{Q}(1) K_{1} \cong \mathbb{Z} / 2\left[c_{4}, c_{6}, c_{8}\right]\left\{1, Q_{0}, Q_{1}\right\}\left\{u_{4}\right\} \\
\bar{Q}(0) K_{0} \cong \mathbb{Z} / 2\left[c_{4}, c_{6}\right] \otimes\left(\mathbb{Z} / 2\left\{u_{3}\right\} \oplus \mathbb{Z} / 2\left\{Q_{2} u_{4}, Q_{3} u_{4}\right\}\right) .
\end{gathered}
$$

The cycle map $\tilde{c l}$ is given by

$$
Q_{0}\left(y^{\prime}\right) \mapsto v_{1} w_{8} \quad, Q_{1} y^{\prime} \mapsto 2 w_{8} \quad, Q_{2} y^{\prime} \mapsto 2 w_{4} w_{8} \quad, Q_{3} y^{\prime} \mapsto 2 c_{8} w_{4} .
$$

Of course the cycle map $\tilde{c l}$ is isomorphic. This fact is still proved by P.Guillot [Gu1], and the case $G=\operatorname{Spin}_{8}$ is computed by Molina [Mo].

$$
\text { 10. } B P^{*}\left(B \operatorname{Spin}_{9}\right) \otimes_{B P^{*}} \mathbb{Z} / 2
$$

At last of this paper, we consider the case $\operatorname{Spin}_{9}$. In [Ko-Ya], we can compute (which is quite complicated)

$$
\begin{gathered}
E\left(\operatorname{Spin}_{9}\right)_{32}^{* * *} \cong B_{4,6,8,16} \otimes\left(B P ^ { * } \left\{1,2 w_{4}, 2 w_{4} w_{8}, 2 w_{8}, v_{1} w_{8},\right.\right. \\
\left.2 w_{4} w_{16}, 2 w_{4} w_{8} w_{16}, 2 w_{8} w_{16}, v_{1} w_{8} w_{16}, 2 w_{16}, v_{1} w_{16} \cdot v_{2} w_{16}\right\} \\
\left.\oplus P(5)^{*}\left[c_{7}\right]\left\{c_{7} c_{8} c_{16}\right\}\right) \oplus B_{4,6,7,8} \otimes P(4)^{*}\left[c_{7}\right]\left\{c_{7} c_{8}\right\} \oplus B_{4,6,16} \otimes P(3)^{*}\left[c_{7}\right]\left\{c_{7}\right\} .
\end{gathered}
$$

This term is the infinite term. (See Theorem 8.3 also.)

Lemma 10.1. ([Ko-Ya])

$$
B P^{*}\left(\text { BSpin }_{9}\right) \otimes_{B P^{*}} \mathbb{Z} / 2 \cong B P^{*}\left(B \operatorname{Spin}_{7}\right) \otimes_{B P^{*}} \mathbb{Z} / 2 \oplus
$$

$\mathbb{Z} / 2\left[c_{4}, c_{6}, c_{8}, c_{16}\right] \otimes\left(\mathbb{Z} / 2\left\{c_{16}, 2 w_{4} c_{16}, 2 w_{4} c_{8} c_{16},\left(2, v_{1}\right) w_{8} c_{16}, 2 w_{4} w_{8} c_{16}\right.\right.$,

$\left.\left.\left(2, v_{1}, v_{2}\right) w_{16}, 2 w_{4} w_{16},\left(2, v_{1}\right) w_{8} w_{16}, 2 w_{4} w_{8} w_{16}\right\} \oplus \mathbb{Z} / 2\left[c_{7}\right]\left\{c_{16} c_{7}\right\}\right)$.

We still know there is an epimorphism from $H^{*}\left(B \operatorname{Spin}_{9} ; H_{\mathbb{Z} / 2}^{*^{\prime}}\right)$ to

$$
\begin{gathered}
H^{*}\left(\text { BSpin }_{7} ; H_{\mathbb{Z} / 2}^{*^{\prime}}\right) \oplus \mathbb{Z} / 2\left[c_{4}, c_{6}, c_{16}\right] \otimes\left(\mathbb{Z} / 2\left[c_{7}\right] \otimes Q(2)\left\{c_{16} u_{3}\right\} \oplus \mathbb{Z} / 2\left\{c_{16} y\right\}\right. \\
\oplus \mathbb{Z} / 2\left[c_{8}, c_{7}\right] \otimes Q(4)\left\{u_{5}\right\} \oplus \mathbb{Z} / 2\left[c_{8}\right] \otimes\left(Q(4) /\left(Q(4)^{+}\right)^{3}\left\{y^{\prime \prime}\right\}\right)
\end{gathered}
$$

where $d_{2} u_{3}=y$ and $d_{2}\left(u_{5}\right)=y^{\prime \prime}$.

We can see the following lemma; 
Proposition 10.2. When $(G, p)=\left(\operatorname{Spin}_{9}, 2\right)$, the cycle map

$$
\tilde{c l}: C H^{*}(B G) / 2 \rightarrow\left(B P^{*}(B G) \otimes_{B P^{*}} \mathbb{Z} / 2\right)
$$

is an epimorphism with $\bmod \left(\mathbb{Z} / 2\left[c_{4}, c_{6}, c_{8}, c_{16}\right]\left\{2 w_{4} w_{16}\right\}\right)$.

The image of the cycle map $\tilde{c l}$ is given as follows. By arguments for $G_{2}$, we see

$$
d_{2}\left(c_{16} u_{3}\right) \mapsto 2 w_{4} c_{16}
$$

If we can see

$$
d_{3}\left(c_{7} u_{5}\right) \mapsto 2 w_{4} w_{16},
$$

then the Totaro's map $\tilde{c l}$ is an epimorphism. Unfortunately, we do not see even $d_{2}\left(c_{7} u_{5}\right)=c_{7} y^{\prime \prime}=0$ yet.

Let us write $Q_{i j}=Q_{i} Q_{j}\left(d_{2} u_{5}\right)=Q_{i} Q_{j}\left(y^{\prime \prime}\right)$. Then the cycle map $\tilde{c l}$ is written as

$$
\begin{aligned}
Q_{01} \mapsto v_{2} w_{16}, & Q_{02} \\
Q_{03} & \mapsto v_{1} w_{8} w_{16}, \quad w_{16}, \quad Q_{13} \mapsto 2 w_{8} w_{16}, \quad Q_{23} \mapsto 2 w_{16}, \\
Q_{04} & \mapsto v_{1} w_{8} w_{16}, \quad Q_{14} \mapsto 2 w_{8} c_{16}, \quad Q_{24} \mapsto 2 w_{4} w_{8} c_{16}, \\
Q_{34} & \mapsto 2 w_{4} c_{8} c_{16} .
\end{aligned}
$$

By the arguments for $\operatorname{Spin}_{7}$, we still know, for example $Q_{1}\left(d_{2} u_{4}\right) \mapsto$ $2 w_{8}$, so we get the $Q_{4} Q_{1}\left(d_{2} u_{5}\right) \mapsto 2 w_{8} c_{16}$ using $Q_{4}\left(u_{5}\right)=c_{16} u_{4}+\ldots$ Similarly we get the maps for $Q_{* 4}$ from that for $\operatorname{Spin}_{7}$.

For other maps, we use the Quillen operation in $B P^{*}(-)$ theory. For a sequence $\alpha=\left(\alpha_{1}, \ldots, \alpha_{m}\right), \alpha_{i} \geq 0$, we have the Quillen cohomology operation in $B P^{*}(X)$ (and also in $A B P^{*}(X)$ ) (see [Ra], [Ha], [Ya2])

$$
r_{\alpha}: B P^{*}(X) \rightarrow B P^{*+|\alpha|}(X) \quad|\alpha|=\sum 2 p^{i} \alpha_{i}
$$

such that $\rho\left(r_{\alpha}\right)=P^{\alpha}$ the fundamental basis of the reduced power operations (see [Ha]) and $r_{\alpha}\left(v_{i}\right) \in \operatorname{Ideal}\left(p, \ldots, v_{i}\right)$. Hence $r_{\alpha}$ acts also on $B P^{*}(X) \otimes_{B P^{*}} \mathbb{Z} / p$.

Let us write by $\bar{S} q^{\text {even }}$ the Quillen operation corresponding $S q^{\text {even }}$. By the definition of $Q_{i}$, se see the equation $S q^{16} S q^{8}\left(Q_{2} Q_{1}\left(u_{5}\right)\right)=$ $Q_{4} Q_{1}\left(u_{5}\right)$ in $H^{*}(B G ; \mathbb{Z} / 2)$. We still know the image of the cycle map

$$
S q^{16} S q^{8}\left(Q_{12}\right)=Q_{14} \mapsto 2 w_{8} c_{16} \in B P^{*}(B G) \otimes_{B P^{*}} \mathbb{Z} / 2 .
$$

Let $\tilde{c l}\left(Q_{12}\right)=x$. Then

$$
\bar{S} q^{16} \bar{S} q^{8}(x)=2 w_{8} c_{16} \quad \text { in } B P^{*}(B G) \otimes_{B P^{*}} \mathbb{Z} / 2 .
$$

So $x$ is non zero. The equation $S q^{16} S q^{8}\left(w_{16}\right)=w_{8} c_{16}$ in $H^{*}(B G ; \mathbb{Z} / 2)$ implies

$$
\bar{S} q^{16} \bar{S} q^{8}\left(2 w_{16}\right)=2 w_{8} c_{16} \bmod \left(v_{1}, \ldots\right) \text { in } B P^{*}(B G) .
$$


Hence we can take $x=2 w_{16}$.

Using $\bar{S} q^{4} \bar{S} q^{2}$ and dimensional reason, we have the first map $Q_{01} \mapsto$ $v_{2} w_{16}$. The other cases are also proved similarly.

The above $Q_{i j}$ are all Griffith elements. From Corollary 9.4, we can write

$$
Q(4)\left\{u_{5}\right\} /\left(Q(4)^{+}\right)^{3}\left\{u_{5}\right\} \cong \oplus_{t=1}^{2} \bar{Q}(t) K_{t}^{\prime} .
$$

In fact, we can take

$$
\begin{gathered}
\bar{Q}(2) K_{2}^{\prime}=\mathbb{Z} / 2\left\{u_{5}, Q_{0}, Q_{1}, Q_{2}, Q_{01}, Q_{02}, Q_{12}\right\}, \\
\bar{Q}(1) K_{1}^{\prime}=\mathbb{Z} / 2\left\{Q_{3}, Q_{03}, Q_{13}, \quad Q_{4}, Q_{04}, Q_{14}\right\}, \\
\bar{Q}(0) K_{0}^{\prime}=\mathbb{Z} / 2\left\{Q_{23}, Q_{24}, Q_{34}\right\} .
\end{gathered}
$$

Recall Corollary 9.4 and $\operatorname{Igr}(W) / \operatorname{Res}_{H \mathbb{Z} / p} \cong \oplus_{t} \bar{Q}(t) K_{t}$. Let $k \in K_{t}$. Then we can identify $k \in H^{*}\left(B G ; H_{\mathbb{Z} / p}^{*^{\prime}}\right)$ and

$$
k(i)=Q_{0} \ldots \hat{Q}_{i} \ldots Q_{t}(k) \in \bar{Q}(t) K_{t} \subset \operatorname{Igr}(W) / \operatorname{Res}_{H \mathbb{Z} / p} .
$$

Moreover suppose $w(k)=t+3$. Since $w(k(i))=3$ and $w\left(d_{r}\right)=1-2 r$, we see

$$
d_{2}(k(i)) \neq 0 \quad\left(\text { hence } d_{2}(k) \neq 0\right) .
$$

Let us consider the projection map

$$
p r .: B P^{*}(X) \otimes_{B P^{*}} \mathbb{Z} / p \rightarrow(9.3) \otimes_{B P^{*}} \mathbb{Z} / p \rightarrow\left(p, v_{1}, \ldots, v_{t}\right)\{\tilde{k}\}
$$

where $\tilde{k}=Q_{0} \ldots Q_{t}(k)$. For $G=G_{2}, \operatorname{Spin}_{7}$ and $\operatorname{Spin}_{9}$, it holds that $\operatorname{pr} . \tilde{c l}\left(d_{2}(k(i))\right)=v_{i} \tilde{k}$, that is,

$$
\operatorname{pr} . \tilde{c l}\left(d_{2}\left(Q_{0} \ldots \hat{Q}_{i} \ldots Q_{t}(k)\right)\right)=v_{i} Q_{0} \ldots Q_{t}(k),
$$

while we do not show it for general cases.

\section{REFERENCES}

[Ad] J. F. Adams. Lectures on exceptional Lie groups, Univ. Chicago Press, Chicago, IL, 1996.

[Bl-Og] S.Bloch and A.Ogus. Gersten's conjecture and the homology of schemes. Ann.Scient.Éc.Norm.Sup. 7 (1974) 181-202.

[Br-Re-Vi] P.Brosman. Z.Reichstein and A.Vistoli. Essential dimension, spinor groups, and quadratic forms. to appear in Ann. Math. (2003).

[Co] J.L.Colliot-Thelene. Cycles algebraiques de torsion et $K$-theorie algebrique. In: Arithmetic algebraic geometry (Tronto, 1991). Lect. Note Math. Springer, Berlin 1553 (1993) 1-49.

[Ga] S.Garibaldi. Cohomological invariants : exceptional groups and Spin groups (wth an appendix by D.Hoffmann). Mem. Amer. Math. Soc. 200. 937 (2009)

[Ga-Me-Se] S.Garibaldi, A.Merkurjev and J.P.Serre. Cohomological invariants in Galois cohomology. University lect. series vol(28) AMS (2003). 
[Gu1] P.Guillot. The Chow ring of $G_{2}$ and $\operatorname{Spin}(7)$. J. Reine angew. Math. 604 (2007) 137-158.

[Gu2] P.Guillot. Geometric methods for cohomological invariants. Document Math. 12 (2007), 521-545.

[Ka] M.Kameko. Poincaré series of cotorsion products. Preprint (2005).

[Ka-Mi] M. Kameko and M. Mimura. Mùi invariants and Milnor operations, Geometry and Topology Monographs 11, (2007), 107-140.

[Ka-Ya1] M.Kameko and N.Yagita. The Brown-Peterson cohomology of the classifying spaces of the projective unitary groups $P U(p)$ and exceptional Lie group. Trans. Amer. Math. Soc. 360 (2008), 2265-2284.

[Ka-Ya2] M.Kameko and N.Yagita. Chern subrings. Proc. Amer. Math. Soc. 138 (2010) 367-373.

[Ko-Ya] A. Kono and N. Yagita. Brown-Peterson and ordinary cohomology theories of classifying spaces for compact Lie groups, Trans. Amer. Math. Soc. 339 (1993), no. 2, 781-798.

[Mo] L.A. Molina. The Chow ring of classifying space of Spins. preprint (2007).

[Mo-Vi] L.Molina and A.Vistoli. On the Chow rings of classifying spaces for classical groups. Rend. Sem. Mat. Univ. Padova 116 (2006), 271-298.

[Or-Vi-Vo] D.Orlov,A.Vishik and V.Voevodsky. An exact sequence for Milnor's Ktheory with applications to quadric forms. Ann. of Math. 165 (2007) $1-13$.

[Pa] W.Paranjape Some spectral sequences for filtered complexes and applications. J. Algebra 186 (1996) 793-806.

[Qu $\quad$ D.Quillen. The mod 2 cohomology rings of extra-special 2-groups and the spinor groups. Math. Ann. 194 (1971), 197-212.

[Re] Z. Reichstein. On the notion of essential dimension for algebraic groups. Transform. groups 5 (2000), 265-304.

[Ro] M.Rost. On the basic correspondence of a splitting variety. preprint (2006)

[Sc-Ya] B. Schuster and N. Yagita, Transfers of Chern classes in BPcohomology and Chow rings, Trans. Amer. Math. Soc. 353 (2001), no. 3, 1039-1054 (electronic).

[Su-Jo] A.Suslin and S.Joukhovistski. Norm Variety. J.Pure and Appl. Algebra 206 (2006) 245-276.

[To] B. Totaro. The Chow ring of classifying spaces. Proc.of Symposia in Pure Math. "Algebraic K-theory" (1997:University of Washington,Seattle) 67 (1999), 248-281.

[Vi] A.Vistoli. On the cohomology and the Chow ring of the classifying space of $P G L_{p}$. J. Reine Angew. Math. 610 (2007) 181-227.

[Vo1] V. Voevodsky. The Milnor conjecture. www.math.uiuc.edu/Ktheory/0170 (1996).

[Vo2] V. Voevodsky. Motivic cohomology with $\mathbb{Z} / 2$ coefficient. Publ. Math. IHES 98 (2003), 59-104.

[Vo3] V. Voevodsky (Noted by Weibel). Voevodsky's Seattle lectures : $K$ theory and motivic cohomology Proc.of Symposia in Pure Math. "Algebraic K-theory" (1997:University of Washington,Seattle) 67 (1999), 283-303. 
[Vo4] V.Voevodsky. Reduced power operations in motivic cohomology. Publ.Math. IHES 98 (2003),1-57.

[Vo5] V.Voevodsky. On motivic cohomology with $\mathbb{Z} / l$-coefficients. www.math.uiuc.edu/K-theory/0631 (2003).

[Ya1] N. Yagita. Examples for the mod p motivic cohomology of classifying spaces. Trans. Amer. Math. Soc. 355 (2003),4427-4450.

[Ya2] N. Yagita. Applications of Atiyah-Hirzebruch spectral sequence for motivic cobordism. Proc. London Math. Soc. 90 (2005) 783-816.

[Ya3] N. Yagita. Coniveau filtration of cohomology of groups. to appear in Proc. London Math. Soc. (2008).

Department of Mathematical Sciences College of Systems EngiNeEring and Science Shibaura Institute of Technology, Saitama, Japan

E-mail address: kameko@shibaura-it.ac.jp

Department of mathematics, Faculty of Science, Ryukyu UniverSITY, OKINAWA, JAPAN

E-mail address: tez@sci.u-ryukyu.ac.jp

Faculty of Education, Ibaraki University, Mito, Ibaraki, Japan

E-mail address: yagita@mx.ibaraki.ac.jp 